\title{
Path-integral Monte Carlo study of the hard-disk solid
}

\author{
P. W. Leung* and G. V. Chester \\ Laboratory of Atomic and Solid State Physics, Cornell University, Ithaca, New York 14853-2501
}

(Received 23 August 1990)

\begin{abstract}
We present a study of the properties of the quantum two-dimensional hard-disk solid at finite temperatures using path-integral Monte Carlo simulations. We performed extensive simulations on systems with $64,144,256,576$, and 1024 disks. We used the image approximation for the hightemperature density matrix and ignored exchange effects. Despite the discontinuous nature of the hard-disk interaction, a long-wavelength harmonic theory provided a good model for the solid. This theory was used as a guide to study the long-range translational and orientational order of the solid at low but finite temperatures where the zero-point motion and finite-temperature excitations are both important. The properties we have computed include the particle displacements, structure factor, angular order, and density of topological defects. We compare these properties with those of the same system in the classical regime and at absolute zero, thus gaining some understanding for the crossover from classical to quantum behavior.
\end{abstract}

\section{INTRODUCTION}

Two-dimensional classical solids have been the subject of extensive computer simulation. Peierls ${ }^{1}$ was the first to point out that two-dimensional solids at finite temperatures do not possess long-range translational order. Within the harmonic phonon picture, he argued that the mean-square displacement of the particles from their lattice sites would diverge as $\ln N$, where $N$ is the system size. Hence, in the thermodynamic limit, no twodimensional crystal can exist. This was proved rigorously by Mermin $^{2}$ using the Bogoliubov inequality. For a solid with long-range translational order, the structure factor $S(G)$ for a reciprocal-lattice vector $\mathbf{G}$ should have a Bragg peak whose height is proportional to $N$. But a two-dimensional solid has a "quasi-Bragg peak" which scales as $N^{1-\eta_{G} / 2}$, where $\eta_{G}$ is positive and lies between zero and one. According to the Kosterlitz-ThoulessHalperin-Nelson-Young ${ }^{6-9}$ (KTHNY) theory of melting, $\eta_{G}$ should have the critical value $\frac{1}{3}$ at the melting point, and is smaller than $\frac{1}{3}$ in the solid phase. The fact that $\eta_{G}$ is nonzero signals the absence of long-range translational order. However, as first pointed out by Mermin, ${ }^{2}$ the long-range orientational order in two-dimensional solids persists and can be used to distinguish between the solid and fluid phases.

In this paper we present a simulation study of the quantum hard-disk solid at finite temperatures. The hard-disk system has been studied in detail in the classical regime. ${ }^{10-12}$ Although the hard-disk potential is purely repulsive, it is generally believed that there is no qualitative difference in the properties of the solid whether the interaction is purely repulsive or has an attractive part, at least in the classical regime. ${ }^{13,14}$ The advantage of working with hard disks lies in the simplicity of the interaction potential, which greatly reduces the computational effort. In fact, it was one of the first systems studied by the Metropolis Monte Carlo method. ${ }^{15}$ Because of the discontinuous nature of the hard-disk potential, a harmonic phonon description is clearly inappropriate and the usual argument about the loss of long-range translational order based on the harmonic model obviously does not apply. In fact, Mermin's argument is inconclusive for the hard-disk potential. However, simulations show the absence of long-range translational order ${ }^{12,16}$ and the presence of long-range orientational order. ${ }^{17}$ We can understand this as follows: in two dimensions the longwavelength phonon modes dominate the thermal behavior when the system size is large, and in this limit the system must behave like an elastic continuum in which small elements of the solid behave as harmonic oscillators. If this argument is correct, we expect the result based on the harmonic model to hold even for the hard-disk system. The standard way to treat the harmonic model in the high-temperature limit (classical regime) is to assume that $k_{B} T / \hbar$ is large compared with all the phonon frequencies. Then $x \equiv \beta \hbar \omega_{\mathrm{qs}}<<1$ for all $\mathrm{q}$ (with $\beta \equiv 1 / k_{B} T$ ) and the phonon number $n_{\mathrm{q} s}$ can be expanded as

$$
\begin{aligned}
n_{\mathrm{q} s} & =\frac{1}{e^{x}-1} \\
& =\frac{1}{x}-\frac{1}{2}+O(x) .
\end{aligned}
$$

The leading term in the expansion gives the temperature dependence in the classical regime. At low temperatures (quantum regime), the low- $|\mathbf{q}|$, low- $\omega$ modes dominate. If the system size is large, then, for these modes, $\beta \hbar \omega_{\mathrm{qs}}$ are small and we can do a similar expansion for them. But since the system is at low temperature, we cannot neglect the zero-point motions which should have a prominent effect on the properties of the system. For example, it has been shown that the ground state of a twodimensional ${ }^{4} \mathrm{He}$ system is a liquid ${ }^{18,19}$ and will solidify only under pressure. This makes the quantum twodimensional systems different from their classical counterparts in two ways. 
(1) The large zero-point motions invalidate the basic assumption of the harmonic model that the particle displacements are small.

(2) The zero-point motions are qualitatively different from the thermal excitations. We may expect the system at zero temperature to possess long-range translational order because the Bogoliubov inequality fails at zero temperature. Furthermore, the long-wavelength excitations which destroy the long-range translational order are absent at zero temperature. Since the zero-temperature behavior is expected to be different, we have an interesting crossover from a state where the thermal phonons dominate the order in the system to a state where the system shows zero-temperature behavior. To study this we need a finite-temperature algorithm, which is the path-integral Monte Carlo (PIMC) method. Previous simulation studies on quantum two-dimensional solids ${ }^{19,20}$ have used the variational or Green's-function Monte Carlo (GFMC) method. Both of these require Gaussian factors to localize the particles ${ }^{21}$ either in the variational wave function or in the importance function which enters the GFMC calculation. This casts doubt on some of the configurational properties such as the mean-square displacement, the singlet distribution function, and the density of topological defects. Using the PIMC method, we can avoid this difficulty since the particles are "free" to move around. In principle, we can go to low enough temperature and extrapolate to zero temperature. In practice, the finite-size effects and slow convergence at low temperatures make this procedure difficult.

The aim of this project is to observe this crossover using the PIMC method at finite temperatures. Extensive simulations have been done on classical two-dimensional solids. ${ }^{9}$ It is now well established from simulations that classical two-dimensional solids do not possess long-range translational order. Young and Alder ${ }^{12}$ showed convincing evidence that the mean-square displacement of a hard-disk solid behaves as $\ln N$, for $N$ in the range $10^{1}-10^{4}$. This result has been reconfirmed and extended to other two-dimensional systems. ${ }^{16}$ The quasi-Bragg peak behaves at a reciprocal-lattice vector $\mathbf{G}$ as ${ }^{17}$

$$
S(G) \sim N^{1-\eta_{G} / 2}
$$

and for $q$ small compared with $G$, as

$$
S(|\mathbf{G}-\mathbf{q}|) \sim q^{-2+\eta_{G}} .
$$

These results provide convincing evidence that twodimensional solids lack long-range translational order. The presence of long-range orientational order is evident from the long-range behavior of the angular correlation function $g_{6}(r)$ defined as

$$
g_{6}(r)=\left\langle e^{6 i[\theta(\mathbf{r})-\theta(0)]}\right\rangle,
$$

where $\theta(\mathbf{r})$ is the bond-angle field. ${ }^{7}$ It should decay to a nonzero constant for large $r$ if the system possesses longrange orientational order. This has been observed in various systems such as the Lennard-Jones ${ }^{22}$ and the inverse 12 -power system. ${ }^{23}$ Our work can provide a bridge between the classical and quantum results. In Sec. II A we give a brief summary of the PIMC formalism and the image approximation for hard disks. In Sec. II B, we formulate our model and show how to calculate various properties of the system in the PIMC formalism. The major problem with our work is that we can only afford to simulate systems a good deal smaller than the classical systems which have been studied. This limits our ability to study the long-range order in the system. Since we cannot do many different system sizes, we have used a long-wavelength harmonic model to analyze our limited data. In Sec. III we develop the harmonic model for the two-dimensional solid. Section IV contains the results of our simulations. First we show that the harmonic model is a good model for the low-temperature properties of the quantum hard-disk solid, we then use it as a guide to analyze our results. In Sec. V, we conclude with a summary of our results and discuss possible future work.

\section{FORMALISM AND MODEL}

\section{A. The path-integral Monte Carlo formalism}

The path-integral Monte Carlo method is a discretized version of Feynman's path-integral method. ${ }^{24}$ The density matrix of a many-body system with Hamiltonian $H$ is a solution to the Bloch equation

$$
\begin{aligned}
& \hbar \frac{\partial \rho(u)}{\partial u}=-H \rho(u), \\
& \rho(0)=\mathbb{1},
\end{aligned}
$$

where $u \equiv \beta \hbar$ is the "imaginary time." Its solution is

$$
\rho(u)=e^{-H u / \hbar}
$$

We can also write the solution as a product of $M$ factors

$$
\begin{aligned}
\rho(u) & =e^{-H u / M \hbar} \cdots e^{-H u / M \hbar} \\
& =\rho\left(\frac{u}{M}\right) \cdots \rho\left(\frac{u}{M}\right) .
\end{aligned}
$$

The significance of Eq. (7) is that the low-temperature density matrix on the left-hand side is broken into a product of high-temperature density matrices, each at a temperature $M / \beta$. In the coordinate representation, Eq. (7) takes the form

$$
\rho\left(R, R^{\prime} ; \beta\right)=\int \cdots \int \prod_{l=1}^{M-1} d R^{l} \rho\left(R, R^{1} ; \beta / M\right) \rho\left(R^{1}, R^{2} ; \beta / M\right) \cdots \rho\left(R^{M-1}, R^{\prime} ; \beta / M\right)
$$


where $R \equiv\left(\mathbf{r}_{1}, \mathbf{r}_{2}, \ldots, \mathbf{r}_{N}\right)$ is the set of particle coordinates of the system. We can interpret this as the sum over the paths formed by $R^{1}, \ldots, R^{M-1}$ which connect $R$ to $R^{\prime}$ in the coordinate space. This is the starting point of the path-integral Monte Carlo formulation. The partition function is given by

$$
Z=\int d R \rho(R, R ; \beta) .
$$

We see that we have to integrate over $M-1$ intermediate configurations $R^{l} \equiv\left(\mathbf{r}_{1}^{l}, \mathbf{r}_{2}^{l}, \ldots, \mathbf{r}_{N}^{l}\right)$. These are called "times slices" since $\beta$ plays the role of imaginary time. $M$ will be called the number of time slices or the Trotter number. ${ }^{25}$ In the picture of the isomorphism between quantum and classical many-body systems, ${ }^{26}$ we can visualize each quantum particle with coordinate $\mathbf{r}_{i}$ as a classical polymer chain with $M$ particles $\left(\mathbf{r}_{i}, \mathbf{r}_{i}^{1}, \mathbf{r}_{i}^{2}, \ldots, \mathbf{r}_{i}^{M-1}\right)$. More explicitly, a quantum two-dimensional $N$-body system is mapped to a stack of $M$ classical two-dimensional $N$-body systems $R, R^{1}, \ldots, R^{M-1}$.

If a high-temperature approximation $\widetilde{\rho}\left(R, R^{\prime} ; \beta / M\right)$ of the density matrix can be found, we can substitute it into the right-hand side of Eq. (8) provided $M$ is large enough to bring $M / \beta$ to the temperature range where the approximation is valid,

$$
\rho\left(R, R^{\prime} ; \beta\right)=\int \cdots \int \prod_{l=1}^{M-1} d R^{l} \widetilde{\rho}\left(R, R^{1} ; \beta / M\right) \cdots \widetilde{\rho}\left(R^{M-1}, R^{\prime} ; \beta / M\right),
$$

and the partition function becomes a multidimensional integral which can be evaluated by the standard Metropolis algorithm. ${ }^{15}$ Note that, although Eq. (8) is exact for any $\beta$ and $M$, substitution of the high-temperature form for the density matrix turns Eq. (10) into an approximation. Although this can be improved by using larger $M$, doing so means larger computational effort. Furthermore, since Eq. (10) is a product of $M$ terms, any error introduced to $\widetilde{\rho}\left(R, R^{\prime} ; \beta / M\right)$ will accumulate giving a larger error in $\rho\left(R, R^{\prime} ; \beta\right)$. So whether this formalism is practical for a given system depends critically on how well we know the asymptotic behavior of the hightemperature density matrix $\widetilde{\rho}$.

The simplest form of the high-temperature approximation is the so-called "primitive approximation," which has been used to study various systems. ${ }^{27}$ It has been shown ${ }^{28}$ that within this approximation, the convergence with increasing $M$ is very unsatisfactory for systems with strongly repulsive interactions such as the Lennard-Jones and hard-disk potentials. Fortunately, for hard spheres (or hard disks), a much better high-temperature approximation exists. For systems with additive pair potentials, we can approximate the high-temperature many-body density matrix by a product of two-body density matrices $^{28-30}$

$$
\begin{aligned}
\widetilde{\rho}\left(R, R^{\prime} ; \beta / M\right)= & \rho^{0}\left(R, R^{\prime} ; \beta / M\right) \\
& \times \prod_{1 \leq i<j \leq N} \frac{\rho_{2}\left(\mathbf{r}_{i}, \mathbf{r}_{j}, \mathbf{r}_{i}^{\prime}, \mathbf{r}_{j}^{\prime} ; \beta / M\right)}{\rho_{1}^{0}\left(\mathbf{r}_{i}, \mathbf{r}_{i}^{\prime} ; \beta / M\right) \rho_{1}^{0}\left(\mathbf{r}_{j}, \mathbf{r}_{j}^{\prime} ; \beta / M\right)},
\end{aligned}
$$

where $\rho^{0}$ is the free-particle density matrix for the $N$ body system, $\rho_{2}$ are the two-body density matrices, and $\rho_{1}^{0}$ are the single-body free-particle density matrices

$\rho^{0}\left(R, R^{\prime} ; \beta / M\right)=\left[\frac{m M}{2 \pi \hbar^{2} \beta}\right]^{N} \exp \left(-\frac{m M}{2 \hbar^{2} \beta} \sum_{i=1}^{N}\left|\mathbf{r}_{i}-\mathbf{r}_{i}^{\prime}\right|^{2}\right)$.

The so-called "image approximation",28,29 provides an analytic expression for $\rho_{2}$ at high temperatures. The idea is that $\rho_{2}$ must satisfy the hard-disk boundary condition, i.e., varnish when two disks touch. It also involves the approximation that the circular boundaries of the disks are approximated by their tangent lines. This should be unimportant at high temperatures where the thermal de Broglie wavelength $\left(\hbar^{2} \beta / m\right)^{1 / 2}$ is small compared to the hard-disk diameter $\sigma$. It can be shown ${ }^{29}$ that, within this approximation,

$$
\frac{\rho_{2}\left(\mathbf{r}_{i}, \mathbf{r}_{j}, \mathbf{r}_{i}^{\prime}, \mathbf{r}_{j}^{\prime} ; \beta / M\right)}{\rho_{1}^{0}\left(\mathbf{r}_{i}, \mathbf{r}_{i}^{\prime} ; \beta / M\right) \rho_{1}^{0}\left(\mathbf{r}_{j}, \mathbf{r}_{j}^{\prime} ; \beta / M\right)}=\left\{\begin{array}{l}
1-\exp \left[-\frac{m M}{\hbar^{2} \beta}\left(r_{i j}-\sigma\right)\left(r_{i j}^{\prime}-\sigma\right)\right] \text { if } r_{i j}, r_{i j}^{\prime}>\sigma, \\
0 \text { if } r_{i j}<\sigma \text { or } r_{i j}^{\prime}<\sigma,
\end{array}\right.
$$

where $m$ is the mass of the disk.

\section{B. Formulation of the model}

We consider a system of hard disks with mass $m$ and diameter $\sigma$. We assume that the particles are distinguishable so that we do not have to consider the exchange effects in implementing the PIMC formalism. We believe that this is a very accurate approximation because we are dealing with the solid phase where exchange is rare. ${ }^{31}$ We shall use reduced units so that $\hbar=m=k_{B}=1$. From now on it is understood that all quantities are actually their respective dimensionless reduced quantities, for example, the density $\rho$ is actually $\rho^{*} \equiv \rho \sigma^{2}$, the temperature $T$ is $T^{*} \equiv k_{B} T /\left(\hbar^{2} / m \sigma^{2}\right)$, and the energy $E$ is $E^{*} \equiv E /\left(\hbar^{2} / m \sigma^{2}\right)$, etc. Putting Eqs. (9)-(13) together, we can write the partition function of the 
hard-disk system as

$$
Z=\int \prod_{l=0}^{M-1} \prod_{i=1}^{N} d \mathbf{r}_{i}^{l} e^{-U_{\mathrm{eff}}}
$$

where

$$
e^{-U_{\mathrm{eff}}} \equiv\left[\frac{M}{2 \pi \beta}\right]^{N M} \exp \left[-\frac{M}{2 \beta} \sum_{l=0}^{M-1} \sum_{i=1}^{N}\left|\mathbf{r}_{i}^{l}-\mathbf{r}_{i}^{l+1}\right|^{2} \prod_{l=0}^{M-1} \prod_{1 \leq i<j \leq N}\left[1-\exp \left[-\frac{M}{\beta}\left(r_{i j}^{l}-1\right)\left(r_{i j}^{l+1}-1\right)\right]\right] .\right.
$$

The simulation cell is a rectangle of aspect ratio $\sqrt{3} / 2$ with periodic boundary conditions. The density and temperature are fixed throughout the simulation $(N-V-T$ ensemble). To improve the efficiency in sampling the phase space, we employ the collective mode moves developed by Runge and Chester ${ }^{32,33}$ in the PIMC study of the hard-sphere system. This code was tested by comparing the pressure with that given by the first-order quantum correction to the classical pressure ${ }^{34}$ and the energy with GFMC calculations. ${ }^{35}$

An estimator for the pressure is

$$
P=\frac{1}{\beta} \frac{\partial \ln Z}{\partial A},
$$

where $A$ is the area of the system. There are two estimators for the kinetic energy. One is obtained by simply differentiating the partition function (the $\beta$ derivative)

$$
K_{\beta}=-\frac{\partial \ln Z}{\partial \beta} \text {. }
$$

The other is the Laplacian estimator

$$
K_{L}=\frac{1}{Z} \int d R\left(\sum_{i=1}^{N}-\left.\frac{1}{2} \nabla_{i}^{2} \rho\left(R, R^{\prime}\right)\right|_{R=R^{\prime}}\right),
$$

where $\nabla_{i}$ operates on the coordinates of particle $i$ of $R$. It is straightforward to derive expressions for $P, K_{\beta}$, and $K_{L}$ from Eq. (14). For those quantities which are diagonal in the coordinate representation, they can be estimated by evaluating them in each time slice and taking the average. These include the mean-square displacement $\left\langle u^{2}\right\rangle$, the structure factor $S(G)$, the long-range angular order $\left|\psi_{N}\right|^{2}$, and the topological defect density.

\section{Effects of finite Trotter number $M$}

The finite-temperature path-integral formalism Eq. (10) holds strictly for $\boldsymbol{M} \rightarrow \infty$ only. At finite $\boldsymbol{M}$ we should be skeptical about the accuracy of Eq. (10). Before we consider the thermodynamic properties, we shall investigate the structural stability of the solid at various $M$. Using a 64-particle system at fixed temperature $T=1.0$ and a particular $M$, we start from a triangular solid and let it equilibrate at a density which we believe to be in the solid phase. The density is then decreased by 0.005 and the system is again equilibrated. This is repeated until the solid melts. The melting of the solid is signaled by a sudden drop in the structure factor evaluated at a reciprocal-lattice vector $G$. The density at which the solid "melts" in this case represents the loss of mechanical stability of the solid, it is not the true thermodynamic melting density. In Fig. 1, the upper end of each bar represents the lowest density where the solid is stable, and the lower end represents the highest density where the solid is seen to melt during the run. At the appropriate densities, the solid usually "melts" after 10 000-20000 Monte Carlo passes. At other higher densities, the system can be run for 40000 passes without melting. We see that the mechanical stability of the system depends on $M$ and, in the present case $(T=1.0), M$ cannot be less than 64 in order to obtain reliable results near the phase-transition region.

At higher densities many-body effects are more important and the two-body density matrix approximation Eq. (11) becomes worse and we have to use larger $M$ which has the effect of pushing the high-temperature density matrices $\widetilde{\rho}$ to a higher temperature. A way to ensure that $M$ is large enough is to calculate the two kinetic-energy estimators Eqs. (16) and (17). They should converge to the same value when $M$ goes to infinity. Following Runge and Chester, ${ }^{32}$ we assume that, for large enough $M$, the kinetic-energy estimators converge as $1 / M$. In Fig. 2 we plot the two kinetic-energy estimators at $\rho=0.45, T=1.0$ and at $\rho=0.475, T=2.0$. All data are averaged over 20000 passes. While within the error limits the two estimators extrapolate to the same value in both cases, the $\beta$ derivative converges more rapidly. We note that, for large enough $M$, the uncertainties in the $\beta$ derivatives are often large enough to include the extrapolated value at infinite $M$. So instead of doing the extrapolation, we can take the $\beta$ derivative as our best estimate for the kinetic energy of the system.

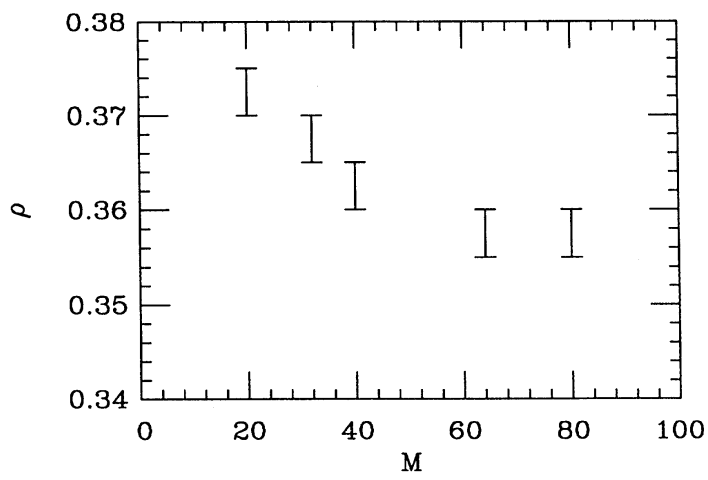

FIG. 1. The mechanical stability of the hard-disk system at $T=1.0$. The upper ends of the bars represent the lowest density where the solid is stable, and the lower ends represent the highest density where the solid seems to melt. 


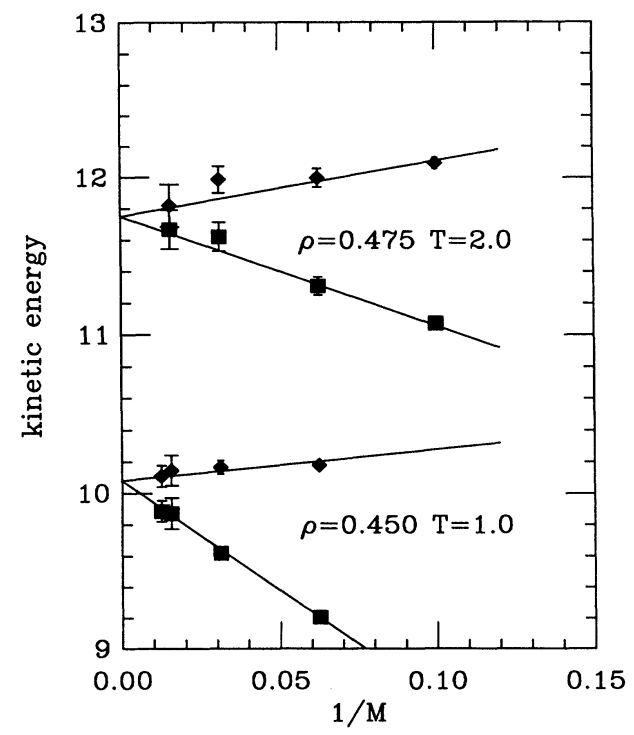

FIG. 2. The convergence of the kinetic-energy estimators with $1 / M$. Laplacian estimators are represented by $\square$ and $\beta$ derivatives are represented by $\downarrow$. The straight lines are guides for the eye.

In Table I we show the variation of the structure factor $S(G)$, the mean-square displacement $\left\langle u^{2}\right\rangle$, and the longrange angular order $\left|\psi_{N}\right|^{2}$ with $M$, all in the solid phase. These structural quantities are all diagonal in the coordinate representation. We see that while the Laplacian estimator requires larger $M$ values for convergence, these diagonal quantities are quite insensitive to the variation in $M$ within the statistical errors ${ }^{36}$ except for the smallest $M$ in each case.

\section{HARMONIC MODEL FOR QUANTUM TWO-DIMENSIONAL SOLID}

For two-dimensional solids, the fluctuations in the long-wavelength modes dominate the thermal behavior in the large- $N$ limit. In this limit, the solid behaves like an elastic continuum and the Debye approximation for the dispersion relation

$$
\omega_{\mathrm{q} s}=c_{\mathrm{q} s} q
$$

holds well since the dominant phonon modes are those near the center of the Brillouin zone. For twodimensional classical solids, the harmonic phonons ${ }^{3-5}$ and the elastic-continuum theory ${ }^{7}$ have been developed. In this section we present a summary of the theoretical results for two-dimensional harmonic solids on a triangular lattice which we will compare with our Monte Carlo results in the later sections. A two-dimensional triangular lattice has the further simplification that it is isotropic: ${ }^{37}$ For any phonon mode with wave vector $q$, there are two branches, one (longitudinal) is polarized along $\mathbf{q}$ and the other (transverse) perpendicular to $\mathbf{q}$. There are only two independent elastic constants, which are usually taken to be the Lamé coefficients $\lambda$ and $\mu$. The longitudinal and transverse sound velocities, which are independent of the direction of $\mathbf{q}$, are given by

$$
\begin{aligned}
c_{l} & =\sqrt{(\lambda+2 \mu) / \rho}, \\
c_{t} & =\sqrt{\mu / \rho} .
\end{aligned}
$$

The longitudinal phonon modes are related to the compression of the system whereas the transverse modes are related to the shearing of the system. In order to compute numerical values for the model, the only quantities needed are the Lamé coefficients. In Sec. III A we present the Monte Carlo evaluation of $\lambda$ and $\mu$ for the quantum hard-disk solid and in Sec. III B, use them to calculate various properties of the model. We emphasize that we are not trying to develop a phonon theory based on particle coordinates for the hard-disk solid, which obviously does not exist. In the elastic continuum limit, the detailed form of the particle interaction is not important. The bulk temperature-dependent properties of the system are solely characterized by the elastic constants, and the purpose of this calculation is to show how accurately we can compute the low-temperature thermal properties of the hard-disk system from the elastic continuum model.

\section{A. Lamé coefficients of the quantum hard-disk solid}

The elastic properties of an isotropic medium are characterized by the Lamé coefficients $\lambda$ and $\mu$. We calculate the shear modulus $\mu$ by applying a finite shear to the system and calculate the resulting stress tensor (see Appendix A). In applying this method one has to make

TABLE I. Variation of the structure factor $S(G)$, mean-square displacement $\left\langle u^{2}\right\rangle$, and long-range angular order $\left|\psi_{N}\right|^{2}$ with $M$. All data are for 64-particle systems and averaged over 20000 passes. All these quantities are diagonal in the coordinate representation.

\begin{tabular}{cccccc}
\hline \hline$\rho$ & $T$ & $M$ & $S(G)$ & $\left\langle u^{2}\right\rangle$ & $\left|\psi_{N}\right|^{2}$ \\
\hline 0.475 & 2.0 & 10 & $25.8 \pm 0.2$ & $0.0866 \pm 0.0009$ & $0.278 \pm 0.003$ \\
& & 16 & $26.4 \pm 0.3$ & $0.0845 \pm 0.0009$ & $0.292 \pm 0.004$ \\
& 32 & $26.5 \pm 0.2$ & $0.0839 \pm 0.0008$ & $0.294 \pm 0.002$ \\
& 64 & $26.5 \pm 0.2$ & $0.0838 \pm 0.0008$ & $0.295 \pm 0.002$ \\
0.450 & & & & \\
& 1.0 & 16 & $24.4 \pm 0.2$ & $0.0980 \pm 0.0006$ & $0.246 \pm 0.002$ \\
& & 62 & $25.5 \pm 0.1$ & $0.0933 \pm 0.0004$ & $0.265 \pm 0.002$ \\
& & $25.2 \pm 0.2$ & $0.0946 \pm 0.0008$ & $0.262 \pm 0.002$ \\
& & $25.3 \pm 0.2$ & $0.0937 \pm 0.0006$ & $0.265 \pm 0.002$ \\
\hline \hline
\end{tabular}


sure that the shear used is small enough so that the stress-strain relation is linear. Since we are dealing with the solid phase away from melting, we can use rather large shears, typically 5\%, without seeing any nonlinear response. Smaller shears are used to make sure that the stress-strain relation is linear. The results are tabulated in Table II.

The simplest way to obtain $\lambda$ is from the isothermal compressibility

$$
\rho\left[\frac{\partial P}{\partial \rho}\right]_{T}=\lambda+\mu \text {. }
$$

$\rho(\partial P / \partial \rho)_{T}$ is estimated by central differencing the pressure evaluated at densities close to the desired density. The results are also tabulated in Table II.

The sound velocities of the solid can now be found by directly substituting the values of $\lambda$ and $\mu$ into Eq. (19). From now on we will assume that the sound velocities are constant over the temperature range we are dealing with, as it is usually done in the Debye model. Another useful quantity is $\bar{c}$ defined by

$$
\frac{1}{\bar{c}^{2}}=\frac{1}{2}\left(\frac{1}{c_{l}^{2}}+\frac{1}{c_{t}^{2}}\right) \text {. }
$$

These are all tabulated in Table II.

\section{B. Properties of the two-dimensional quantum harmonic solid}

In this section we shall compute the low-temperature behavior of those quantities that will be used in later sections. The most common way to deal with the sum over the phonon modes in the harmonic model is to replace it by an integral. In this way the temperature and $N$ dependence for the quantities can be found in the large- $N$ limit.

It is straightforward to show that the internal energy of a quantum harmonic solid is given by ${ }^{38}$

$$
E(T)=\sum_{\mathbf{q}, s} \omega_{\mathbf{q} s}\left(n_{\mathbf{q} s}+\frac{1}{2}\right) .
$$

The first term is the finite-temperature contribution and the second term is the zero-point energy. For the finitetemperature term, at very low-temperature the longwavelength (low- $|\mathbf{q}|)$ modes are the only excited modes and we can replace the sum by an integral. Using the Debye approximation Eq. (18) and defining the twodimensional Debye temperature ${ }^{39}$ as

$$
\Theta_{D}=2 \sqrt{\pi \rho} \bar{c}
$$

where $\bar{c}$ is given by Eq. (21), one finds that the internal energy per particle is given by

$$
\frac{E(T)}{N}=\frac{8 \zeta(3)}{\Theta_{D}^{2}} T^{3}+\frac{E(0)}{N}, \quad \zeta(3)=1.20205,
$$

where we have written the zero-temperature term as $E(0)$. This is the well-known $T^{3}$ law for the energy in two dimensions. Direct substitution of the data in Table II into Eq. (23) gives $\Theta_{D}=19.0$ at $\rho=0.45$ and $\Theta_{D}=22.0$ at $\rho=0.475$.

The corresponding result for the mean-square displacement is

$$
\left\langle u^{2}(T)\right\rangle=\frac{1}{N} \sum_{\mathbf{q}, s} \frac{1}{\omega_{\mathrm{q} s}}\left(n_{\mathrm{q} s}+\frac{1}{2}\right) .
$$

In the classical limit we expand the exponent using Eq. (1) and replace the sum by an integral. In two dimensions this gives a logarithmic divergence in $N$. This is the first indication of the loss of long-range translational order in classical two-dimensional solids. ${ }^{1}$ As argued before, we can do a similar expansion at low temperatures. It differs from the high-temperature expansion in that it is only done for the low- $\omega$ modes. Since the low- $\omega$ modes dominate the finite-temperature part, we can replace its sum by an integral and expand $n_{\mathrm{q} s}$. For the zero-temperature term, we write it as $\left\langle u^{2}(0)\right\rangle$. Equation (25) then becomes

$$
\left\langle u^{2}(T)\right\rangle=\frac{T}{2 \pi \bar{c}^{2} \rho} \ln N+a(T)+\left\langle u^{2}(0)\right\rangle,
$$

where $a(T)$ is that part of the finite-temperature term which is independent of $N$. At absolute zero, we expect the system to have long-range translational order and $\left\langle u^{2}(0)\right\rangle$ to behave like

$$
\left\langle u^{2}(0)\right\rangle=a^{\prime}-\frac{b^{\prime}}{\sqrt{N}} .
$$

For large $N$, the finite-temperature term in Eq. (26) eventually takes over and $\left\langle u^{2}(T)\right\rangle$ will depend on $N$ logarithmically. The fact that the $\ln N$ term in Eq. (26) goes to zero as $T$ goes to zero demonstrates the return of the long-range translational order.

A direct measure of the long-range translational order is the structure factor evaluated at a reciprocal-lattice vector. In the harmonic model, it is given by ${ }^{40,4}$

$$
S(G)=\sum_{i=1}^{N} \exp \left[-\frac{G^{2}}{2 N} \sum_{\mathbf{q}, s} \frac{1}{\omega_{\mathbf{q} s}}\left(n_{\mathbf{q} s}+\frac{1}{2}\right)\left[1-\cos \left(\mathbf{q} \cdot \mathbf{R}_{i}\right)\right]\right] \text {. }
$$

In the large- $N$ limit, the structure factor has a quasiBragg peak ${ }^{4,7}$

$$
S(G) \sim N^{1-\eta_{G} / 2},
$$

where

TABLE II. Lamé coefficients and sound velocities for 64-particle systems in the solid phase. The temperature is at $T=1.0$ and $M=80$.

\begin{tabular}{cccccc}
\hline \hline$\rho$ & $\mu$ & $\lambda+\mu$ & $c_{l}$ & $c_{t}$ & $\bar{c}$ \\
\hline 0.450 & $18.2 \pm 0.6$ & 51.4 & 12.4 & 6.4 & 8.0 \\
0.475 & $23.7 \pm 0.4$ & 83.9 & 15.1 & 7.1 & 9.0 \\
\hline \hline
\end{tabular}




$$
\begin{aligned}
\eta_{G} & =\frac{G^{2}}{2 \pi \rho \bar{c}^{2}} T \\
& =\frac{G^{2}}{4 \pi} \frac{3 \mu+\lambda}{\mu(2 \mu+\lambda)} T
\end{aligned}
$$

Again, the dependence of $\eta_{G}$ on $T$ indicates the return of the long-range translational order as $T$ goes to zero. At absolute zero, $S(G) \sim N$.

Although two-dimensional solids have no long-range translational order in the thermodynamic limit, they can be distinguished from the fluid phase by the presence of long-range orientational order. We consider a bond orientational order parameter for the triangular lattice ${ }^{7}$ $\psi(\mathbf{r})$ defined as

$$
\psi(\mathbf{r})=e^{6 i \theta(r)}
$$

where $\theta(\mathbf{r})$ is the angle which the bond at $\mathbf{r}$ make with an arbitrary axis. The long-range angular order for the system, defined as ${ }^{16}$

$$
\left|\psi_{N}\right|^{2}=\left\langle\left|\frac{1}{N} \sum_{i=1}^{N} \psi\left(\mathbf{r}_{i}\right)\right|^{2}\right\rangle
$$

is given by (see Appendix B)

$\left|\psi_{N}\right|^{2}=\frac{1}{N} \sum_{i=1}^{N} \exp \left[-\frac{9}{N} \sum_{\mathbf{q}} \frac{q^{2}}{\omega_{\mathbf{q} t}}\left(n_{\mathbf{q} s}+\frac{1}{2}\right)\left[1-\cos \left(\mathbf{q} \cdot \mathbf{R}_{i}\right)\right]\right]$.

It is interesting to note that, in Eq. (33), only the transverse phonon modes contribute. This is because a uniform compression of the whole system will not change the bond orientational order. The long-range orientational order of the system manifests itself through the large- $N$ behavior of $\left|\psi_{N}\right|^{2}$. In Appendix B we show that $\left|\psi_{N}\right|^{2}$ goes to a constant independent of $N$ for very large $N$.

All the results we have obtained are only valid for sufficiently large $N$. For intermediate values of $N$ we cannot replace the sum over phonon modes by an integral. To overcome this difficulty we have constructed a "discrete mode model" in which the sum is done over all the allowed phonon modes in the first Brillouin zone. By carrying out this sum in Eqs. (25), (28), and (33) we are able to know how important the finite-size effects are and for what values of $N$ the elastic continuum limit can be expected to hold. The discrete mode calculations for $\left\langle u^{2}\right\rangle, S(G)$, and $\left|\psi_{N}\right|^{2}$ are shown in Figs. 3-5, respectively. From them we infer that $N$ has to be in the order of 1000 to reach the elastic continuum limit. Therefore, we have to be careful about the finite-size effects when we analyze our simulation results.

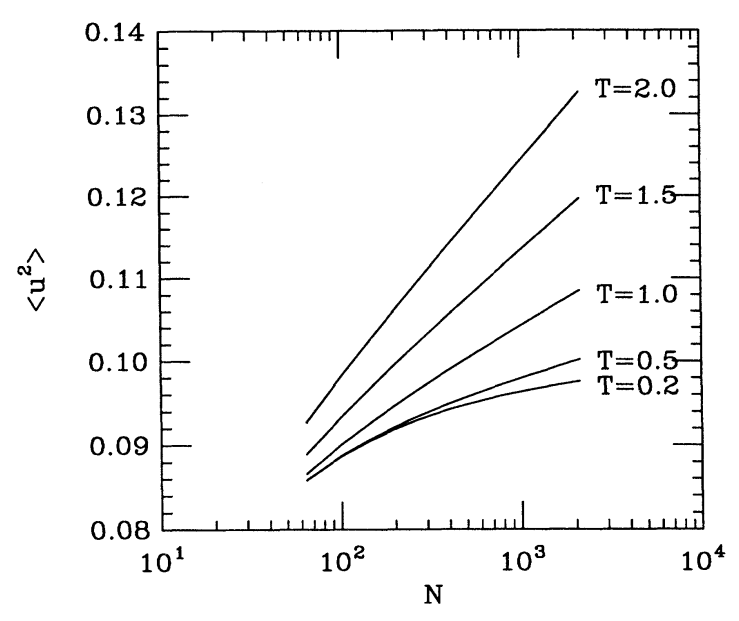

FIG. 3. The size dependence of $\left\langle u^{2}\right\rangle$ at $\rho=0.45$ and different temperatures calculated from the harmonic model.

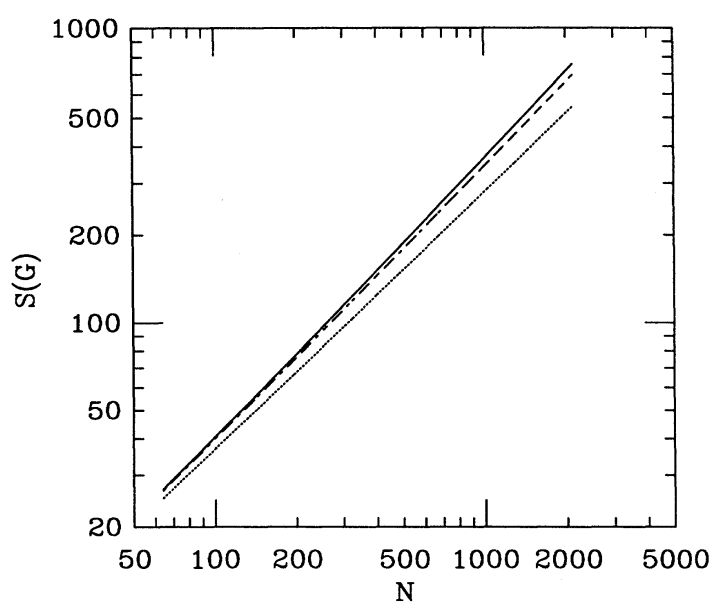

FIG. 4. The size dependence of $S(G)$ at $\rho=0.45$ and different temperatures calculated from the harmonic model. Meanings for the lines are $T=0.5(-), T=1.0(---)$, and $T=2.0$ $($....).

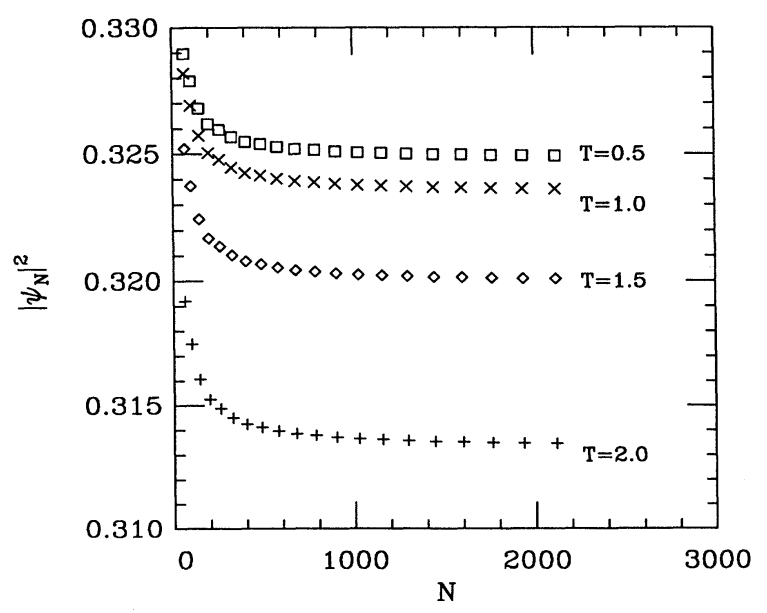

FIG. 5. The size dependence of the long-range angular order at different temperatures calculated from the harmonic model. 


\section{RESULTS OF THE SIMULATION}

In this section we present the simulation results for the hard-disk triangular solid. The system sizes are usually 64,144 , and 256 particles. For those quantities that depend strongly on the system size, we have also simulated a 576-particle system in order to get more information on the size dependence. Typical run lengths are from 20000 to 40000 passes. Unlike classical hard disks, the quantum mechanics introduces a temperature scale for the quantum hard disks. We can calibrate this temperature scale by mapping the hard-disk system onto a twodimensional ${ }^{4} \mathrm{He}$ system. ${ }^{41}$ Using the Aziz potential ${ }^{42}$ and integrating the Schrödinger equation, ${ }^{43}$ we find the $s$-wave scattering length in two dimensions to be $2.15 \AA$. If we take this to be the hard-disk diameter $\sigma$, then a reduced temperature equal to 1 corresponds to $2.6 \mathrm{~K}$ for the ${ }^{4} \mathrm{He}$ system. Our lowest temperature, 0.5 , thus corresponds to $1.3 \mathrm{~K}$ for the ${ }^{4} \mathrm{He}$ system.

To locate the melting density accurately, one has to do a free-energy computation for both the solid and fluid phases. But since we do not have the exchange implemented in our code, our fluid-phase calculations can only be approximately correct. We therefore did not do a free-energy analysis. Instead, we located the melting density $\rho_{m}$ roughly by doing a Maxwellian construction in the melting region in the $P$ versus $\rho$ diagram. At $T=1.0$, $\rho_{m}$ is found to be about 0.37 and at $T=2.0, \rho_{m}$ is about 0.44 . These are to be compared with the classical melting density $\rho_{m}=0.912$ (Ref. 10 ) and the close-packed density $\rho_{\mathrm{CP}}=1.1547$. It is clear that the melting density of the quantum solid is much lower and this allows more freedom for each particle. These densities can be compared with the melting densities of other systems at zero temperature, for example, $\rho_{m}=0.44$ (Ref. 44) and $\rho_{m}=0.33$ (Ref. 20) for the two-dimensional ${ }^{4} \mathrm{He}$ system with Lennard-Jones and Aziz interactions, respectively. ${ }^{45}$ Our work mainly concentrates on the two isochores $\rho=0.45$ and 0.475 . At $\rho=0.45$, the system is deep in the solid phase at $T=1.0$ and close to melting at $T=2.0$, whereas at $\rho=0.475$, it is far from the melting region at all temperatures that we considered. In the calculations with system sizes $N=64,144,256$, and 576 , we use $M=64$ when the system is close to melting, i.e., $\rho=0.45$ and $T=2.0$, and $M=80$ for the higher-density solids. The adequacy of these $M$ values are tested by observing the difference between the two kinetic-energy estimators and by performing test runs at other $\boldsymbol{M}$ values. Note that 80 is also the largest $M$ value used by Runge and Chester ${ }^{32}$ for their highest-density hard-sphere solid. Based on the observation in Sec. III C, we used $M=32$ for system size $N=1024$, since we wish to compute those quantities which are diagonal in the coordinate representation. For such quantities, small- $M$ values suffice.

\section{A. Monte Carlo cooling from the fluid phase}

It is known that, in the classical two-dimensional onecomponent system interacting with a pair potential, the triangular solid forms readily by quenching from the disordered fluid phase and no glassy state can be ob- tained. ${ }^{46}$ It is interesting to see if the quantum solid can be formed as readily from the fluid. We equilibrated two systems of 64 and 256 quantum hard disks, respectively, at $\rho=0.45$ and $T=5.0$. Then the temperature was suddenly decreased to $T=1.0$. By observing the contour plots of the distribution of particles in both systems, we found that a triangular solid is readily formed in the 256particle system after about 10000 Monte Carlo passes. In the 64-particle system, some frozen-in defects are obtained because the system cannot align with the boundary of the simulation cell. We believe this is a finite-size effect. We conclude from this that the quantum solid is readily formed by quenching from the fluid phase, as in the classical case.

\section{B. Kinetic energy}

In Sec. III B it was shown that when the temperature is low enough so that the long-wavelength phonon modes dominate the thermal behavior, the energy of the system should show a $T^{3}$ dependence as in Eq. (24). The kinetic energies found from our simulations at $\rho=0.45$ for various temperatures in the solid phase are plotted in Fig. 6(a). Using a linear least-squares fitting, we find the Debye temperature

$$
\Theta_{D}=18.6 \pm 2.1 \text {. }
$$
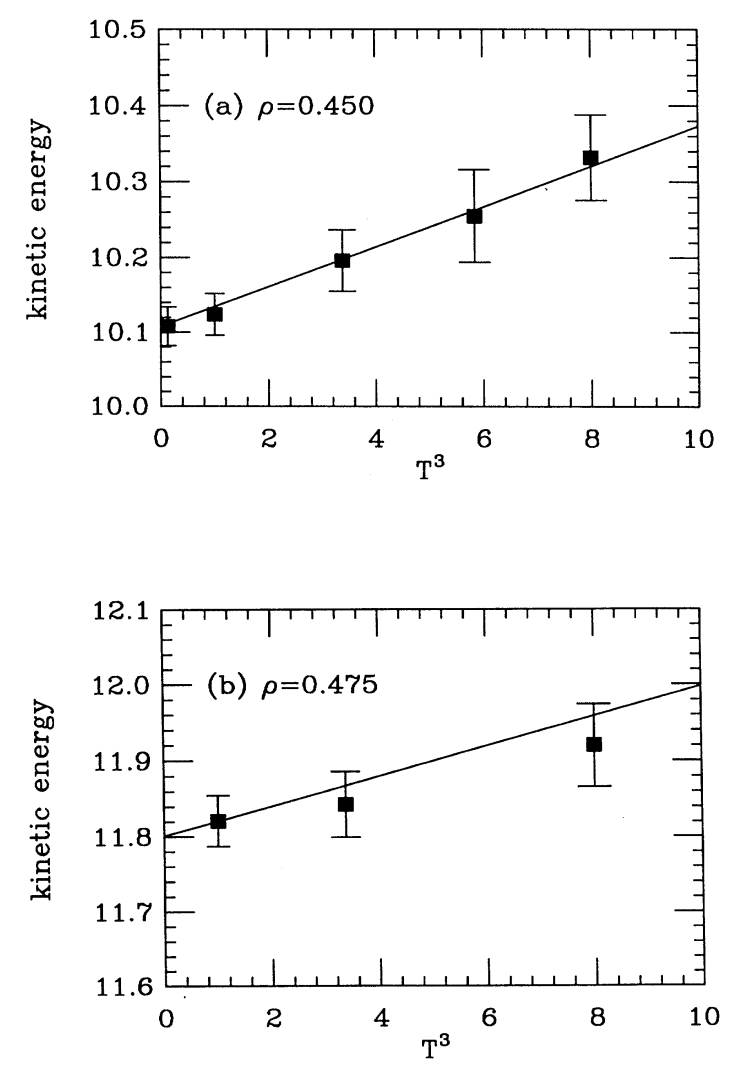

FIG. 6. The kinetic energy as a function of $T^{3}$ at densities (a) $\rho=0.450$ and (b) $\rho=0.475$. The straight lines are from the harmonic model with the zero-point energies properly corrected (see text). 
Although the uncertainty is quite large, this value agrees very well with Eq. (23) which gives $\Theta_{D}=19.05$. For the zero-point energy $E(0)$, we do not use the prediction from the harmonic theory

$$
E(0)=\frac{1}{2} \sum_{\mathbf{q}, s} \omega_{\mathbf{q} s},
$$

because this sum is dominated by the high- $\omega$ modes. The large zero-point motion also violates the basic assumption for the harmonic model. Therefore, in order to compare the simulation results with the predictions of the harmonic model, we need the zero-point energy $E(0)$. From Fig. 6(a) we see that, at the lowest temperature $(T=0.5)$, the energy is essentially indistinguishable from $E(0)$ within the error limit. So we can substitute the energy at $T=0.5$ for $E(0)$ in Eq. (24) and perform the discrete mode calculation for the energies at different temperatures. These are plotted as a straight line in Fig. 6(a). Again we see that the agreement with the simulation results is very good. We conclude that, in spite of the small system sizes we have used, the behavior of our system is very close to the elastic continuum limit. We also remark that the entropy of the system can be determined from the slope of Fig. 6(a). The corresponding results at $\rho=0.475$ are shown in Fig. 6(b). The agreement in this case does not look as good as the lower density one. But we also note that the change in the kinetic energy over the temperature range is very small and it is difficult to pin down the variation of the kinetic energy with temperature precisely in this case.

\section{Mean-square displacement}

The mean-square displacement of one particular configuration is calculated by averaging over the meansquare displacements of each individual time slice. In classical simulations, $\left\langle u^{2}\right\rangle$ is easy to determine for highdensity solids but shows large fluctuations near melting, or is even ill defined. ${ }^{16}$ Although we are now dealing with systems deep in the solid phase ${ }^{47}$ due to the low densities, $\left\langle u^{2}\right\rangle$ is not so well behaved as in the classical case. Long "time" fluctuations show up. This makes it difficult to obtain good estimates for $\left\langle u^{2}\right\rangle$ for a manageable run length. Moreover, our runs for the quantum system are much shorter than for the classical systems that have been simulated.

It is well known that the mean-square displacement of the classical hard-disk system exhibits a logarithmic divergence with system size, ${ }^{12,16}$ which is what we expect from the long-wavelength phonon modes. From Sec. IV $B$ we see that, in the temperature range in which we are working, the thermal energy is, at most, a few percent of the total energy. In other words the system has large zero-point motions with very little thermal excitation. But as Sec. II B shows, the $\ln N$ divergence of the thermal excitations will eventually dominate at large enough $N$. The simulated mean-square displacement for various system sizes $N$ are plotted in Fig. 7. Although we expect the zero-temperature term $\left\langle u^{2}(0)\right\rangle$ in Eq. (26) to have some functional dependence on $N$, with the limited data we have, we can do no better than fitting to a simple $\ln N$ dependence for $\left\langle u^{2}(T)\right\rangle$,

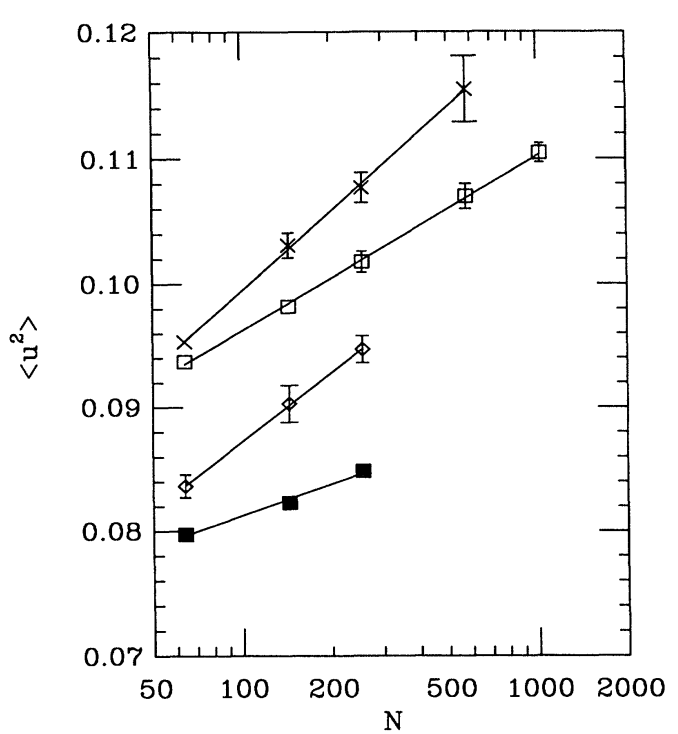

FIG. 7. The size dependence of the mean-square displacements from simulation. The meanings of the symbols are $(X)$ $\rho=0.45$ and $T=1.5$, ( $\square) \rho=0.45$ and $T=1.0,(\diamond) \rho=0.475$ and $T=2.0$, and ( $\square) \rho=0.475$ and $T=1.0$. The straight lines are the best fit to the data assuming that they follow Eq. (36).

$$
\left\langle u^{2}(T)\right\rangle=A+B \ln N .
$$

In other words, we assume that the size dependence of $\left\langle u^{2}\right\rangle$ is dominated by the finite-temperature part. Table III shows the coefficients in Eq. (36) obtained by linear least-squares fitting. In Fig. 8 , we plot the coefficient $B$ versus the temperature. Based on the harmonic theory, we expect $B$ to depend linearly on $T$ [see Eq. (26)]

$$
B=\frac{1}{2 \pi \bar{c}^{2} \rho} T .
$$

This relation is shown as straight lines for the two densities in Fig. 8. It is quite convincing that our results fit the expected trend, which shows the return of the long-range translational order as $T$ goes to zero.

Making a quantitative comparison is not as straightforward as in the previous subsection. And since we are plotting $\left\langle u^{2}\right\rangle$ versus $N$, we cannot ignore the size dependence of $\left\langle u^{2}(0)\right\rangle$. The simplest way to get around this is to subtract it off by finding the difference of $\left\langle u^{2}\right\rangle$ at two temperatures. These are readily found in the harmonic model and are plotted as a solid line in Fig. 9 together with the simulated data. Again we see the agreement in the finite-temperature part of $\left\langle u^{2}\right\rangle$, although the error

TABLE III. The coefficients $A$ and $B$ in (36) found by linear least-squares fitting to the data in Fig. 7.

\begin{tabular}{cccc}
\hline \hline$\rho$ & $T$ & $A$ & $B$ \\
\hline 0.450 & 1.0 & $0.068 \pm 0.002$ & $0.0061 \pm 0.0003$ \\
& 1.5 & $0.058 \pm 0.003$ & $0.0091 \pm 0.0007$ \\
0.475 & 1.0 & $0.065 \pm 0.002$ & $0.0036 \pm 0.0005$ \\
& 2.0 & $0.051 \pm 0.005$ & $0.0080 \pm 0.0010$ \\
\hline \hline
\end{tabular}




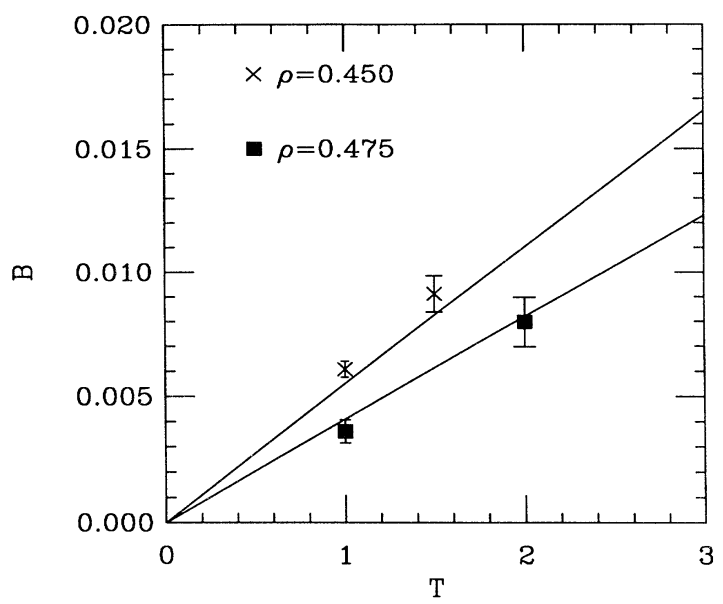

FIG. 8. The coefficient $B$ as defined in Eq. (36) as a function of $T$. The straight lines are predictions from the harmonic model as in Eq. (37).

limits in our simulated data are large.

The above analysis seems to show that the mean-square displacement at low temperatures behaves very much like that in the classical regime since, in both cases, $\left\langle u^{2}\right\rangle$ behaves like Eq. (36). But in the classical regime, the zero-point motion is ignored and the coefficient $A$ comes from the finite-temperature contribution only, which is $a(T)$ in Eq. (26). In the present case, $A$ contains both the finite-temperature part $a(T)$ and the zero-temperature part $\left\langle u^{2}(0)\right\rangle$ as in Eq. (26). A comparison with Table III in Ref. 12 reveals that the zero-point motions in the present case dominate the magnitude of the mean-square displacement. ${ }^{48}$ The coefficients $A$ and $B$ are of the same order of magnitude in the classical regime whereas $A$ is much larger than $B$ in the quantum regime. This means that the finite-temperature part in the quantum regime is contributing much less than its classical counterpart. The mean-square displacement at low temperatures are

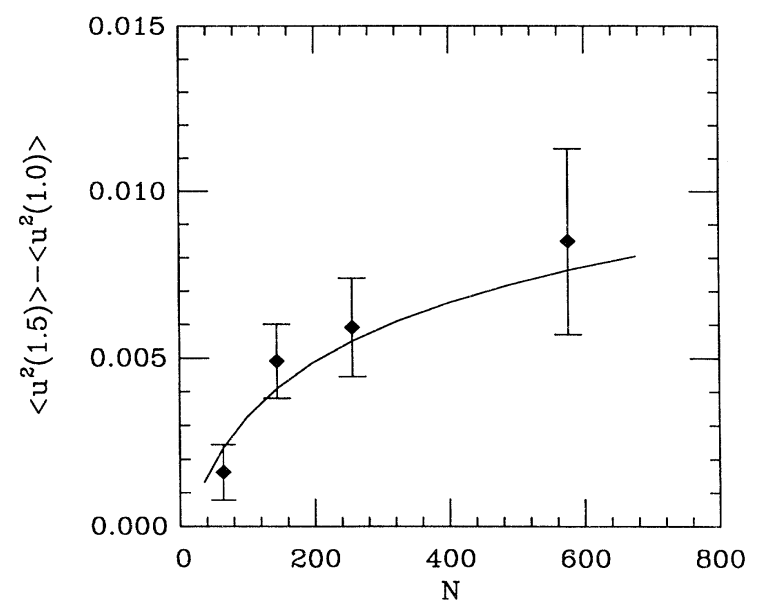

FIG. 9. The difference of the mean-square displacements at two temperatures $T=1.0$ and 1.5 at $\rho=0.45$ as a function of system size $N$. The solid line is the prediction of the harmonic model. large compared with classical systems. This is also reflected in the comparatively small values of $S(G)$ and $\left|\psi_{N}\right|^{2}$ as shown later.

\section{Singlet distribution function}

The singlet distribution function $P(R)$ is defined such that $P(R) 2 \pi R d R$ is the probability of finding a particular particle inside a circular shell at a distance $R$ from its lattice site. In the harmonic model, $P(R)$ is a Gaussian. ${ }^{49}$ In two dimensions, the ratio of its moments $\left\langle u^{4}\right\rangle / 2\left\langle u^{2}\right\rangle^{2}$ is a measure of the deviation of $P(R)$ from a Gaussian. This ratio is exactly 1 if $P(R)$ is a Gaussian. In Table IV we show the ratio $\left\langle u^{4}\right\rangle / 2\left\langle u^{2}\right\rangle^{2}$ at various temperatures for the 256-particle system at $\rho=0.45$. When the system is well inside the solid phase $(T=1.0$ and 1.5), we find no deviation from Gaussian within experimental error. This is consistent with variational and Green's function Monte Carlo studies of two-dimensional quantum solids. ${ }^{20}$ However, in our simulations we do not need to use localization factors for the particles. This result shows that the behavior of $P(R)$ is very consistent with the harmonic model even when the zero-point motions dominate. Another very important result should be valid. Since

$$
\left\langle u^{4}\right\rangle \simeq 2\left\langle u^{2}\right\rangle^{2},
$$

and $\left\langle u^{2}\right\rangle$ grows as $\ln N$ for large $N$, the fluctuation in $\left\langle u^{2}\right\rangle$

$$
\delta u^{2}=\left(\left\langle u^{4}\right\rangle-\left\langle u^{2}\right\rangle^{2}\right)^{1 / 2} \sim \ln N
$$

increase as $N$ increases, which makes it more difficult to get a good estimate for $\left\langle u^{2}\right\rangle$ as we go to larger systems.

When the system is near melting $(T=2.0), P(R)$ has a secondary peak at around one lattice spacing as shown in Fig. 10. A plot of the configuration reveals that two particles have exchange positions. This behavior has also been observed in the classical hard-disk systems ${ }^{50}$ and is believed to be the cause of the continuous rise in $\left\langle u^{2}\right\rangle$ near melting. ${ }^{16}$ Once this occurs, $\left\langle u^{2}\right\rangle$ is ill defined. This is the reason why the data at $\rho=0.45$ and $T=2.0$ is missing from Fig. 7. We also note that the primary peak in $P(R)$ is still accurately a Gaussian as shown in Fig. 10, although we expect nonlinear effects to set in near melting.

\section{E. The structure factor}

An estimator for the structure factor is

$$
S(G)=\left\langle\frac{1}{M N} \sum_{l=0}^{M-1}\left|\sum_{j=1}^{N} e^{i \mathrm{G} \cdot \mathbf{r}_{j}^{l}}\right|^{2}\right\rangle \text {. }
$$

TABLE IV. The ratio $\left\langle u^{4}\right\rangle / 2\left\langle u^{2}\right\rangle^{2}$ for 256 -particle systems at $\rho=0.45$.

\begin{tabular}{cc}
\hline$T$ & $\left\langle u^{4}\right\rangle / 2\left\langle u^{2}\right\rangle^{2}$ \\
\hline 1.0 & $0.999 \pm 0.001$ \\
1.5 & $0.9998 \pm 0.0005$ \\
2.0 & $3.15 \pm 0.03$ \\
\hline
\end{tabular}




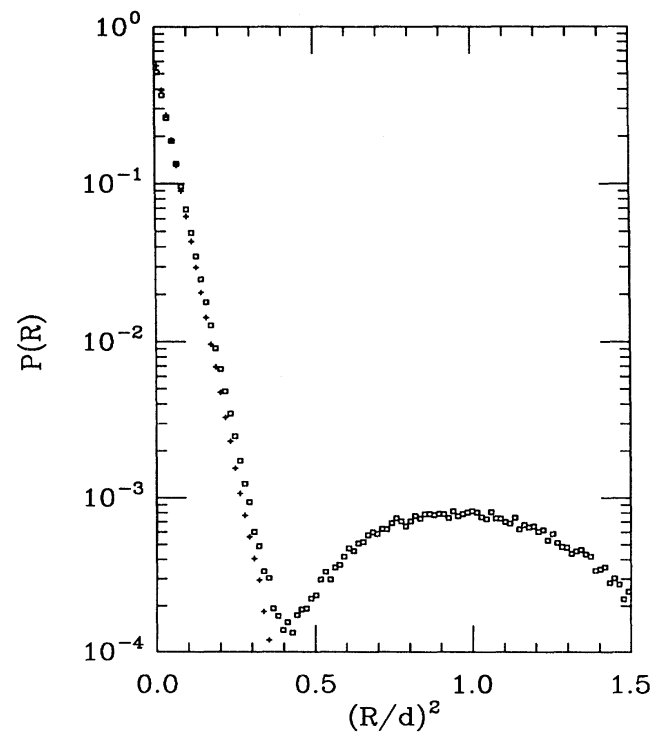

FIG. 10. The singlet distribution function at $\rho=0.45$ and $T=1.0(+)$ and $T=2.0(\square) . d$ is the nearest-neighbor distance.

We calculate it by averaging the results of evaluating Eq. (40) over the three reciprocal-lattice vectors $4 \pi / \sqrt{3} d(0,1), 4 \pi / \sqrt{3} d\left( \pm \sqrt{3} / 2, \frac{1}{2}\right)$, where $d$ is the lattice constant. Besides being used to indicate the mechanical stability of the solid (see Sec. II C), the structure factor also measures the long-range translational order of the solid. This has been carefully studied in several classical systems. ${ }^{17}$ In Fig. 11 we plot $S(G)$ versus systems size at various temperatures and densities. We find that $S(G)$ behaves like a quasi-Bragg peak [see Eq. (29)]. From the slope of the plot, we can find $\eta_{G}$ according to Eq. (29). The results for $\rho=0.45$ are listed in Table $V$ and plotted in Fig. 12. As mentioned in Sec. III B, $\eta_{G}$ is expected to go to zero as $T$ goes to zero. This signals the return of long-range translational order. But we note that the two data points at lower temperatures show systematic deviations. We believe that these are aue to finite-size effects. Equation (29) holds only in the large- $N$ limit. Furthermore, careful inspection of the discrete mode calculation for $S(G)$ in Fig. 4 shows that $\ln S(G)$ versus $\ln N$ is not exactly a straight line. It bends slightly upward at small $N$. If we simply fit a straight line to a few points at small $N$, we will obtain a slope smaller than the true slope at large $N$ which give $\eta_{G}$ through Eq. (29) and hence overestimate $\eta_{G}$. This will be more serious when we go to lower temperatures.

If we assume a low-temperature expansion for the structure factor,

$$
\frac{S(G)}{N}=\frac{S(G, T=0)}{N}+\frac{S^{\prime}(G, T>0)}{N},
$$

and if we believe that the harmonic model is a good description for the finite temperature-behavior of our systems, we can find $S(G, T=0)$ by fitting Eq. (41) to the simulation results, with $S^{\prime}(G, T>0)$ given by the temperature-dependent part of Eq. (28). Figure 13 shows such a fit at $\rho=0.45$ and for $N=144,256,576$, and 1024 .
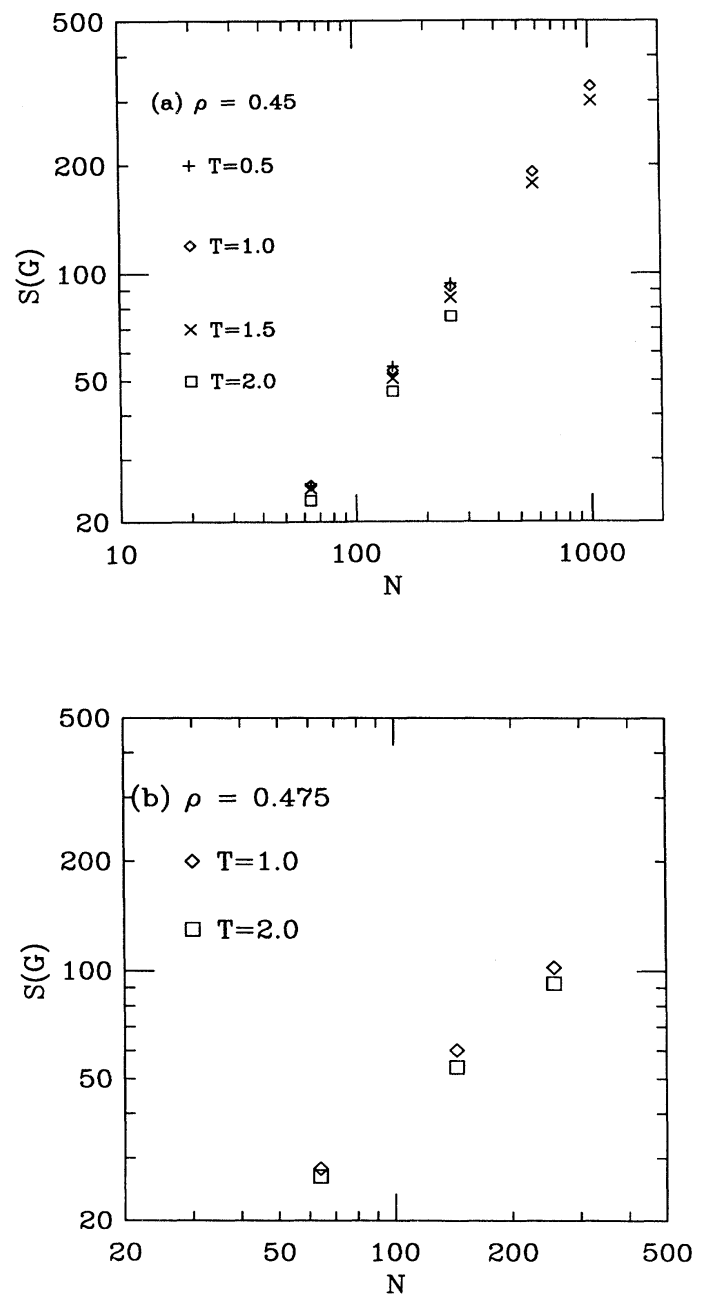

FIG. 11. The size dependence of the structure factor at different temperatures at (a) $\rho=0.450$ and (b) $\rho=0.475$.

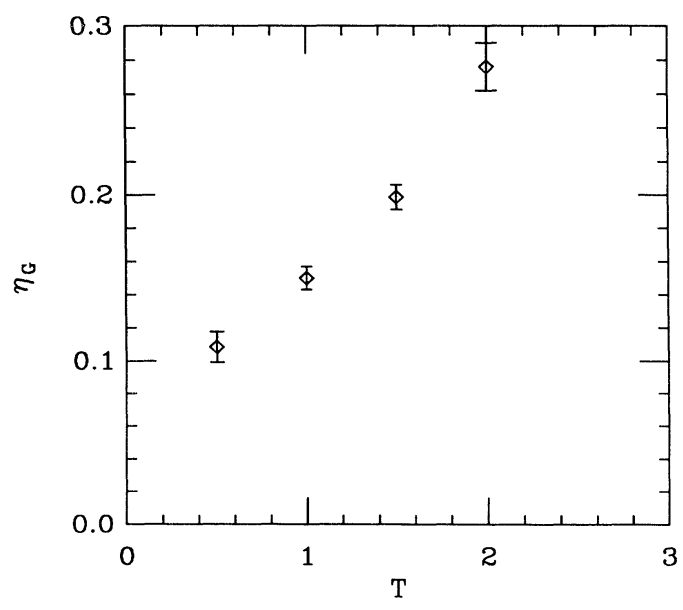

FIG. 12. $\eta_{G}$ as a function of $T$ at density $\rho=0.45$. 
TABLE V. $\eta_{G}$ at $\rho=0.45$ and 0.475 .

\begin{tabular}{ccc}
\hline \hline$\rho$ & $T$ & $\eta_{G}$ \\
\hline 0.450 & 0.5 & $0.108 \pm 0.009$ \\
& 1.0 & $0.150 \pm 0.007$ \\
& 1.5 & $0.199 \pm 0.007$ \\
& 2.0 & $0.276 \pm 0.014$ \\
0.475 & & \\
& 1.0 & $0.13 \pm 0.01$ \\
\hline \hline
\end{tabular}

Note that, at $T=2.0$, the simulation results fall below the values given by $\mathrm{Eq}$. (41) because they are probably too close to melting. We infer from Fig. 13 that $S(G, T=0) / N$ converges to a constant as $N$ increases, which will mean that at $T=0$, the system will have longrange translational order.

When compared with the classical hard-disk system, $S(G)$ in the present case is smaller. At a density $\rho=0.923$ (about $1 \%$ away from melting), $S(G)$ for a 256 classical hard-disk system is about 141.7 , compared with 91.8 for a 256 quantum hard-disk system at $\rho=0.45$ and $T=1.0$ (roughly $20 \%$ away from melting). This means that quantum solid shows much less positional order than its classical counterpart.

\section{F. Long-range angular order parameter}

Starting from the definition of the long-range angular order $\left|\psi_{N}\right|^{2}$, Eq. (32),

$$
\begin{aligned}
\left|\psi_{N}\right|^{2} & =\left\langle\left|\frac{1}{N} \sum_{i=1}^{N} \psi\left(\mathbf{r}_{i}\right)\right|^{2}\right\rangle \\
& =\frac{1}{N} \sum_{i=1}^{N}\left\langle\psi\left(\mathbf{r}_{i}\right) \psi(0)\right\rangle \\
& =\frac{1}{N} \sum_{i=1}^{N} g_{6}\left(r_{i}\right),
\end{aligned}
$$

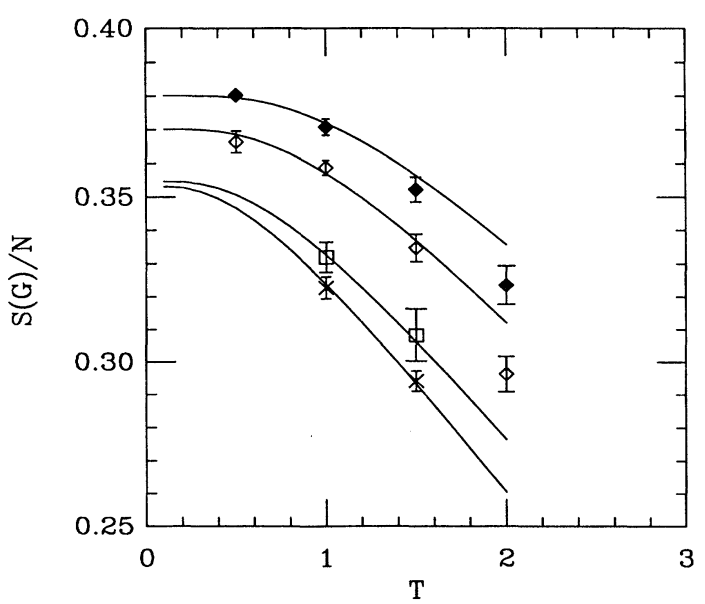

FIG. 13. The fit of the simulation data on the structural factor at $\rho=0.45$ using Eq. (41) to find the zero-temperature values. The meanings of the symbols are $(\diamond) N=144,(\diamond)$ $N=256$, ( $\square) N=576$, and $(\times) N=1024$. where $g_{6}(r)$ is the angular correlation function. It is well known that, in the solid phase, $g_{6}(r)$ goes to a constant for large $r$. The behavior of $g_{6}(r)$ (Ref. 22) is, in fact, very similar to $\left|\psi_{N}\right|^{2}$ as shown in Fig. 5. Since the sum in Eq. (42) is dominated by the large $r_{i}$ 's, $\left|\psi_{N}\right|^{2}$ goes to a constant at very large $N$. This is essentially the same conclusion as in Sec. III B.

In our simulation, we evaluate $\psi(\mathbf{r})$ as defined in (31) by

$$
\psi\left(\mathbf{r}_{i}\right)=\frac{1}{n_{i}} \sum_{\substack{j=1 \\ \text { NN }}}^{n_{i}} e^{6 i \theta_{i j}},
$$

where the sum is over the nearest neighbors (NN) of particle $i, n$ is the number of nearest neighbors, and $\theta_{i j}$ is the orientation of the nearest-neighbor bond between particles $i$ and $j$ relative to an arbitrary axis. Following Zollweg, ${ }^{51}$ we define the nearest neighbors of a particle as those within a distance of $\sqrt{7 / \pi \rho}$ from that particle. This distance is chosen so that, on the average, each particle has six nearest neighbors. The expression for $\left|\psi_{N}\right|^{2}$ then becomes

$$
\left|\psi_{N}\right|^{2}=\left\langle\left|\frac{1}{N} \sum_{i=1}^{N} \frac{1}{n_{i}} \sum_{\substack{j=1 \\ \text { NN }}}^{n_{i}} e^{6 i \theta_{i j}}\right|^{2}\right\rangle .
$$

An estimator for $\left|\psi_{N}\right|^{2}$ is obtained by averaging Eq. (44) over the time slices. The results are plotted in Fig. 14. They all show a decreasing trend with increasing system sizes. A comparison with Fig. 5 reveals that our system sizes are too small for $\left|\psi_{N}\right|^{2}$ to reach a constant. However, the similarity between Figs. 14 and 5 suggests that $\left|\psi_{N}\right|^{2}$ does approach a constant for large $N$ and that there is long-range orientational order in the quantum solid.

When compared with the classical hard-disk system,

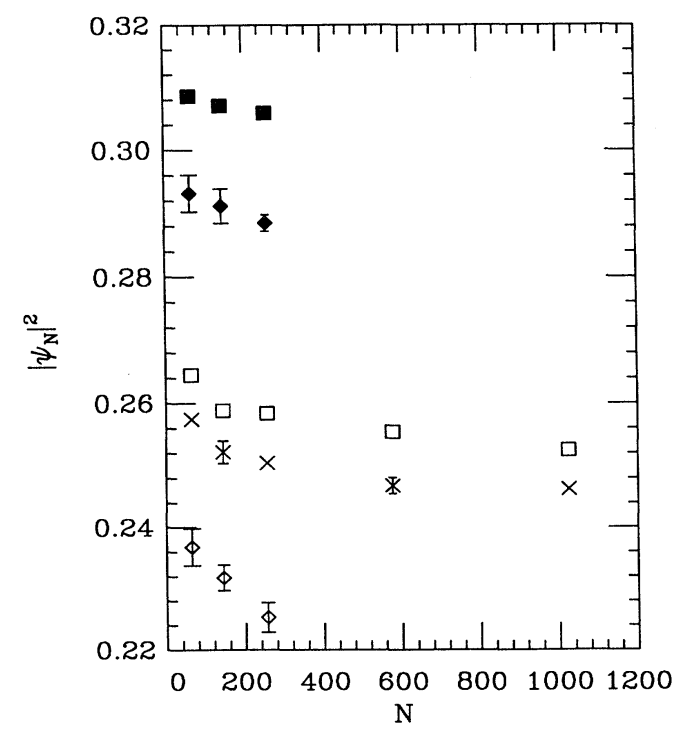

FIG. 14. The size dependence of the long-range angular order. The meanings of the symbols are (E) $\rho=0.475$ and $T=1.0,(\diamond) \rho=0.475$ and $T=2.0$, ( $\square) \rho=0.450$ and $T=1.0$, $(\times) \rho=0.450$ and $T=1.5$, and $(\diamond) \rho=0.450$ and $T=2.0$. 


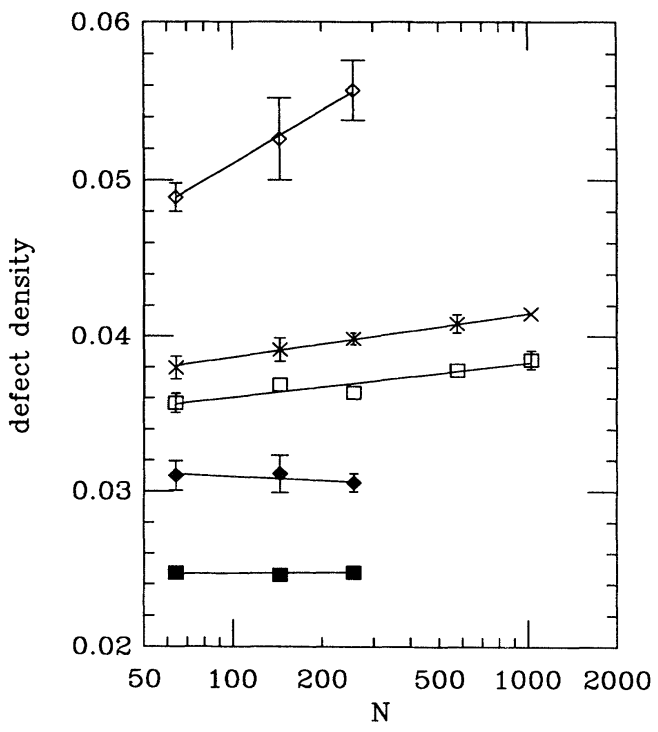

FIG. 15. The size dependence of the defect density. The meanings of the symbols are (ם) $\rho=0.475$ and $T=1.0,(\checkmark)$ $\rho=0.475$ and $T=2.0$, (口) $\rho=0.450$ and $T=1.0,(\times) \rho=0.450$ and $T=1.5$, and $(\diamond) \rho=0.450$ and $T=2.0$. The lines are the best straight line fits to the data.

$\left|\psi_{N}\right|^{2}$ in the present case is much smaller. Even when it is close to melting (about $1 \%$ away in density), the classical hard-disk solids have a $\left|\psi_{N}\right|^{2}$ value of about 0.6 (Ref. 16) whereas the present values of $\left|\psi_{N}\right|^{2}$ range from about
0.2 to 0.3 even for systems which are far away from melting. This means that the quantum solid shows less longrange angular order than its classical counterpart.

\section{G. Topological defects}

We study the topological defects in two dimensions by carrying out the standard Voronoi polygon construction. ${ }^{52}$ The defect density is defined to be $\left(N_{5}+2 N_{4}\right) / N$, where $N_{5}$ and $N_{4}$ are the number of five- and four-sided polygons, respectively. This calculation is done for each of the time slices and the average is taken to be the estimate of the defect density. The primary importance of studying the topological defects in two dimensions is its relation with the melting mechanism. Here we are interested in knowing the effects of the topological defects on the structural order of the solid.

As in classical simulations, the main difficulty in studying the defect density is that it is difficult to obtain good statistics, since the number of defects is small unless we have a very large system. The results are plotted in Fig. 15. It immediately reveals the familiar fact that the defect density increases with the system size when the system is close to melting. However, the topological defects are usually understood to be thermally excited and, as pointed out before, the thermal energies of our systems are, at most, a few percent of the total energies. It is not clear whether this increase in defect density is a thermal effect or is a nonlinear effect when the system is close to melting. If it is because of the latter reason, we should be

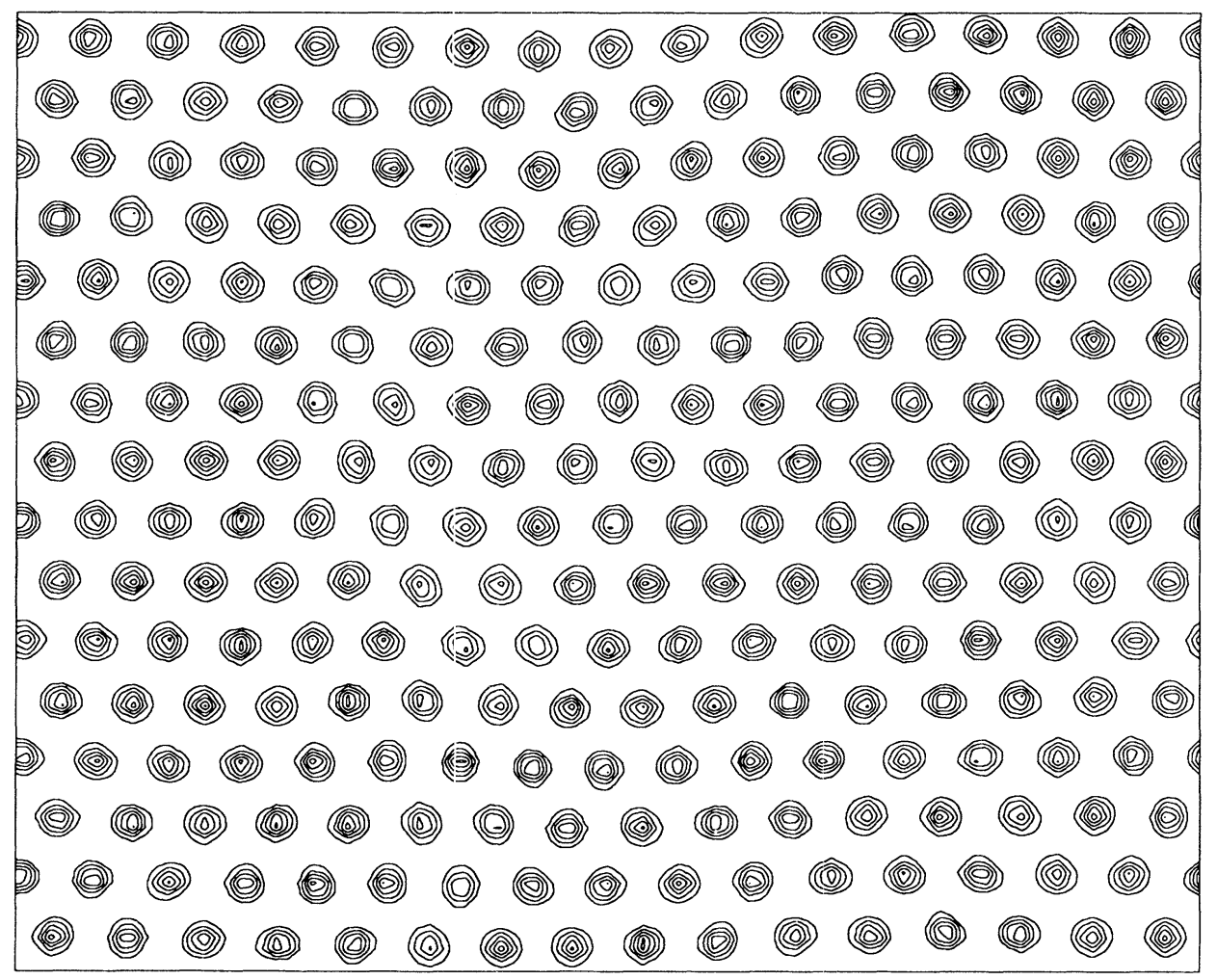

FIG. 16. A contour plot of the particle distribution at $\rho=0.450$ and $T=1.0$ for the 256-particle system. 
TABLE VI. Size dependence of the defect density at different temperatures and densities.

\begin{tabular}{ccrc}
\hline \hline$\rho$ & $T$ & $N$ & Defect density \\
\hline 0.450 & 1.0 & 64 & $0.0357 \pm 0.0006$ \\
& & 144 & $0.0369 \pm 0.0005$ \\
& & 576 & $0.0364 \pm 0.0004$ \\
& & 1024 & $0.0378 \pm 0.0004$ \\
& & 64 & $0.0385 \pm 0.0006$ \\
& 1.5 & 144 & $0.0380 \pm 0.0008$ \\
& & 576 & $0.0391 \pm 0.0008$ \\
& & 1024 & $0.0398 \pm 0.0004$ \\
& & 64 & $0.0415 \pm 0.0006$ \\
& & 144 & $0.049 \pm 0.001$ \\
& 2.0 & 256 & $0.053 \pm 0.003$ \\
& & 64 & $0.056 \pm 0.002$ \\
& & 144 & $0.0247 \pm 0.0003$ \\
& & 256 & $0.0246 \pm 0.0003$ \\
& & 64 & $0.0248 \pm 0.0001$ \\
& & 144 & $0.0310 \pm 0.0003$ \\
& & 256 & $0.0311 \pm 0.0012$ \\
& & & $0.0306 \pm 0.0006$ \\
\hline \hline
\end{tabular}

able to observe it even at zero temperature.

There is a major difference between the present result and those of the classical system. While the defect density of a classical hard disk near melting is only around $0.007,{ }^{16}$ our defect densities are several times larger than this even for high-density solids. For the purpose of comparison, we tabulate the data in Table VI. In Fig. 16 we show a contour plot for the probability distribution of the system at $\rho=0.45$ and $T=1.0$. It certainly shows a very ordered solid. Figure 17 shows the topological defects in two of the time slices after 26000 passes. Most of the dislocations are bound together either in pairs or in clusters. However, as has been pointed out by Strandburg, ${ }^{9}$ it is difficult to give a clear interpretation from the defect map for small systems. But in our case, the higher defect density clearly suggests that topological defects can be formed more readily in the quantum regime. But the orderliness of the system as shown in Fig. 16 shows that the topological defects are very short lived and may not have a prominent effect on the stability of the solid.

\section{CONCLUSION}

In the previous sections we showed that the finitetemperature behavior of the kinetic energy of the quantum hard-disk solid follows a $T^{3}$ law, the mean-square displacement diverges as $T \ln N$ when $N$ is large enough, and the singlet distribution function is very accurately a Gaussian. We conclude from these observations that the harmonic model provides a good description for the finite-temperature behavior of the quantum hard-disk solid. This reveals the importance of the long-wavelength elastic excitations. We further show that the harmonic model can provide reasonably good quantitative agreements for the low-temperature thermal properties with the elastic moduli obtained from simulation. We then study the long-range translation and orientational order at different temperatures. Although our results at the lower temperatures are not very reliable due to finite-size effects and slow convergence, we showed that, at higher temperatures, their behavior agrees well with the predictions from the harmonic model. By assuming the same functional dependence on $T$ as given by the harmonic model, we extrapolate these two quantities to zero temperatures and show that they agree with the prediction that the long-range translational order reappears as the temperature approaches zero. The study of the longrange angular order $\left|\psi_{N}\right|^{2}$ seemed to show that longrange orientational order exists in the quantum solid at any temperature. Finally, our studies on the topological defects show that the systems possess large defect densities at low temperatures, even at the high-density solid

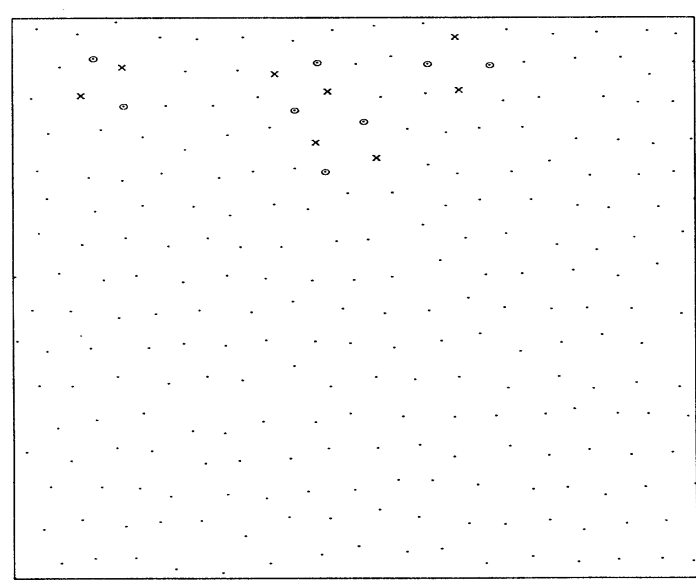

(a)

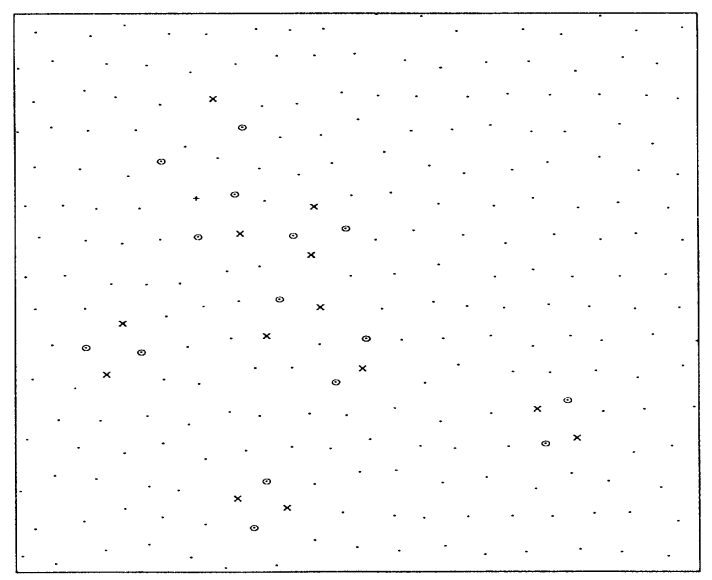

(b)

FIG. 17. Maps of the topological defects of the 256-particle system at $\rho=0.450$ and $T=1.0$ after 26000 passes. The locations of the particles are marked by $(\cdot)$. Particles with five neighbors are marked by $(O)$, with seven neighbors marked by $(X)$ and with eight neighbors marked by $(+)$. 
phase. Extrapolation seemed to suggest that there is still a large number of defects at zero temperature. However, they are very short lived so that they can be "washed out" by averaging over a period of run as in Fig. 16.

When compared with the classical hard-disk systems, the major difference lies in the structural order of the systems. The quantum hard-disk system remains as a solid at very low densities compared with the classical harddisk solid. Although the quantum solids are stable in the densities and temperatures we have simulated, the smaller values of $S(G)$ and $\left|\psi_{N}\right|^{2}$ show that they possess weaker long-range translational and orientational order as compared with the classical solid.

We still have some problems to solve. The primary limitations of the present work are the small system sizes and short run times with which we can deal. Both of which are very small when compared with the classical hard-disk systems. For the classical system one can afford 1000000-2 000000 Monte Carlo passes for system sizes up to tens of thousands of particles. ${ }^{53}$ The data we can generate for these systems are very much more accurate than for the quantum system. At present, our data are quite noisy and longer running times are needed to improve the statistics. The size dependence of $S(G)$, $\left\langle u^{2}\right\rangle$, and $\left|\psi_{N}\right|^{2}$ and the defect density can be made more convincing if we have data for larger system sizes. Furthermore, we need to go to lower temperatures if we want to get reliable results at zero temperature by extrapolation. All these require a major increase in computing time. When the system is close to melting, we see an increase in the defect density with system size. This is well known in classical systems but since we do not know whether this is a result of the long-wavelength fluctuation or a nonlinear effect near melting, we cannot speculate on whether this will happen to the system close to melting at zero temperature. It may be instructive to study the system at absolute zero. The recently developed shadow wave function, ${ }^{54}$ which does not have localization factors, is a strong candidate for this purpose.

\section{ACKNOWLEDGMENTS}

We would like to thank K. J. Runge for helpful discussions and for providing his quantum hard-sphere code, K. E. Schmidt for communicating his unpublished GFMC calculations on the quantum hard disks, and J. A. Zollweg for providing information on the classical harddisk system. This work is supported by the National Science Foundation (NSF) under Grant No. DMR-8718983. We also acknowledge the computer time allocations from the Cornell National Supercomputer Facility, a resource of the Center for Theory and Simulation in
Science and Engineering of Cornell University, which is funded in part by the NSF, New York State and the IBM Corporation and members of the Corporate Research Institute, and from the National Center for Supercomputing Applications of the University of Illinois at UrbanaChampaign, which is funded by the NSF.

\section{APPENDIX A: SHEAR MODULUS FOR THE QUANTUM HARD DISK}

In this appendix we describe a method to calculate the shear modulus $\mu$ for a quantum two-dimensional solid. This is directly adapted from the method of Broughton et al. ${ }^{55}$ for the classical solids. It has the advantage of producing data with smaller fluctuations and so is more accurate than the other methods. ${ }^{56}$

In this method, we apply a small shear to the $x$ direction

$$
\begin{aligned}
& x^{\prime} \rightarrow x+\epsilon_{x y} y, \\
& y^{\prime} \rightarrow y,
\end{aligned}
$$

and so the displacement vector is

$$
\begin{aligned}
\mathbf{u} & =\mathbf{r}^{\prime}-\mathbf{r} \\
& =\left(\epsilon_{x y} y, 0\right) .
\end{aligned}
$$

The strain tensor $u_{i j}$, defined as

$$
u_{i j}=\frac{1}{2}\left(\frac{\partial u_{i}}{\partial x_{j}}+\frac{\partial u_{j}}{\partial x_{i}}\right),
$$

is

$$
\begin{aligned}
& u_{x x}=u_{y y}=0, \\
& u_{x y}=u_{y x}=\frac{1}{2} \epsilon_{x y} .
\end{aligned}
$$

In an isotropic medium, the free energy of the strained system is given in terms of the Lamé coefficients by ${ }^{37}$

$$
\begin{aligned}
F & =F_{0}+\frac{1}{2} \lambda \sum_{i} u_{i i}^{2}+\mu \sum_{i k} u_{i k}^{2}+\cdots \\
& =F_{0}+\mu\left(u_{x y}^{2}+u_{y x}^{2}\right)+\cdots .
\end{aligned}
$$

Thus, the stress tensor $\sigma_{x y}$ is

$$
\begin{aligned}
\sigma_{x y} & =\frac{\partial F}{\partial u_{x y}} \\
& =2 \mu u_{x y}+O\left(u_{x y}^{2}\right) \\
& =\mu \epsilon_{x y}+O\left(\epsilon_{x y}^{2}\right)
\end{aligned}
$$

By differentiating Eq. (14) we get

$$
\begin{aligned}
\sigma_{x y}=\frac{\rho}{\beta N} & \frac{M^{M}}{\beta} \sum_{l=0}^{M-1} \sum_{i=1}^{N}\left(x_{i}^{l}-x_{i}^{l+1}\right)\left(y_{i}^{l}-y_{i}^{l+1}\right) \\
& \left.-\frac{M}{\beta} \sum_{l=0}^{M-1} \sum_{1 \leq i<j \leq N} \frac{\exp \left(-\frac{M}{\beta}\left(r_{i j}^{l}-1\right)\left(r_{i j}^{l+1}-1\right)\right]}{1-\exp \left[-\frac{M}{\beta}\left(r_{i j}^{l}-1\right)\left(r_{i j}^{l+1}-1\right)\right]}\left[\left(r_{i j}^{l+1}-1\right) \frac{x_{i j}^{l} y_{i j}^{l}}{r_{i j}^{l}}+\left(r_{i j}^{l}-1\right) \frac{x_{i j}^{l+1} y_{i j}^{l+1}}{r_{i j}^{l+1}}\right]\right) \epsilon_{x y},
\end{aligned}
$$


where $\langle\cdots\rangle_{\epsilon_{x y}}$ is the ensemble average in the strained system. $\mu$ is then determined from the linear term in the stress-strain relation (A5).

To evaluate the ensemble average in (A6), we transform the equilibrated system into a strained system through (A1). The simulation cell then becomes a parallelogram with the periodic boundary conditions modified to

$$
\left(x+\epsilon_{x y} L_{y}, y+L_{y}\right)==(x, y) .
$$

In applying this method, we must always remember to make sure that the shear $\epsilon_{x y}$ used is small so that (A5) maintains its linear form. If the shear is too large, a nonlinear response will set in and the value of $\mu$ determined from the linear form of (A5) will decrease. We repeat the calculation with different values of the shear to make sure that (A5) is linear. Whenever possible, larger shears are preferred because they reduce the fluctuations in the ensemble average.

\section{APPENDIX B: LONG-RANGE ANGULAR ORDER IN THE HARMONIC THEORY}

In this appendix we derive Eq. (33) and study its large$N$ behavior. From the harmonic theory, the displacement of particle $i$ from its lattice site $\mathbf{R}_{i}$ is

$$
\begin{aligned}
\mathbf{u}_{i} & \equiv \mathbf{r}_{i}-\mathbf{R}_{i} \\
& =\frac{1}{\sqrt{2 N}} \sum_{\mathbf{q}, s} \frac{1}{\sqrt{\omega_{\mathbf{q} s}}} \epsilon_{\mathbf{q} s}\left(a_{\mathbf{q} s} e^{i \mathbf{q} \cdot \mathbf{R}_{i}}+a_{\mathbf{q} s}^{\dagger} e^{-i \mathbf{q} \cdot \mathbf{R}_{i}}\right),
\end{aligned}
$$

where $\epsilon_{\mathrm{q} s}$ are the polarization vectors, which are unit vectors pointing along (longitudinal) or perpendicular (transverse) to the direction of propagation $\mathbf{q}$. The bond- angle field, which is the angle the nearest-neighbor bond at $\mathbf{r}$ makes with an arbitrary axis, is

$$
\theta(\mathbf{r})=\frac{1}{2}|\nabla \times \mathbf{u}(\mathbf{r})| \text {. }
$$

From Eqs. (31) and (32)

$$
\begin{aligned}
\left|\psi_{N}\right|^{2} & =\frac{1}{N}\left\langle\sum_{i=1}^{N} e^{6 i\left[\theta\left(\mathbf{r}_{i}\right)-\theta(0)\right]}\right\rangle \\
& =\frac{1}{N} \sum_{i=1}^{N} e^{-18\left\langle\left[\theta\left(\mathbf{r}_{i}\right)-\theta(0)\right]^{2}\right\rangle} .
\end{aligned}
$$

Consider the term in the exponent, by using Eq. (B1)

$$
\begin{aligned}
\left\langle\left[\theta\left(\mathbf{r}_{i}\right)-\theta(\mathbf{0})\right]^{2}\right\rangle= & \frac{1}{4}\left\langle\left|\nabla \times\left[\mathbf{u}\left(\mathbf{r}_{i}\right)-\mathbf{u}(0)\right]\right|^{2}\right\rangle \\
= & \frac{1}{2 N} \sum_{\mathbf{q}} \frac{q^{2}}{\omega_{\mathbf{q} t}}\left(\left\langle a_{\mathbf{q} t}^{\dagger} a_{\mathbf{q} t}\right\rangle+\frac{1}{2}\right) \\
& \times\left[1-\cos \left(\mathbf{q} \cdot \mathbf{R}_{i}\right)\right] .
\end{aligned}
$$

Substituting into Eq. (B3), we get Eq. (33).

In the large- $N$ limit, we substitute Eq. (1) into Eq. (B4)

$$
\left\langle[\theta(\mathbf{r})-\theta(\mathbf{0})]^{2}\right\rangle=\frac{1}{4 \pi \rho \beta c_{t}^{2}} \frac{1}{R^{2}} \int_{0}^{q_{D} R} d x x\left[1-J_{0}(x)\right],
$$

where $q_{D}$ is the Debye wave vector (independent of $N$ ) and $J_{0}$ is the Bessel function of zeroth order. $\left|\psi_{N}\right|^{2}$ is dominated by the large- $R$ behavior of (B5):

$$
\left\langle[\theta(\mathbf{r})-\theta(\mathbf{0})]^{2}\right\rangle \simeq \frac{1}{8 \pi \rho \beta c_{t}^{2}} q_{D}^{2},
$$

which is independent of $N$. Hence, in the large- $N$ limit, $\left|\psi_{N}\right|^{2}$ is a constant independent of $N$.
*Present address: Supercomputer Computations Research Institute, Florida State University, Tallahassee, Florida 323064052 .

${ }^{1}$ R. E. Peierls, Helv. Phys. Acta 7, 81 (1923); Ann. Inst. Henri Poincare 5, 177 (1935).

${ }^{2}$ N. D. Mermin, Phys. Rev. 176, 250 (1968).

${ }^{3}$ B. Jancovici, Phys. Rev. Lett. 19, 20 (1967).

${ }^{4}$ H. J. Mikeska and H. Schmidt, J. Low Temp. Phys. 2, 371 (1970).

${ }^{5}$ Y. Imry, CRC Crit. Rev. Solid State Mater. Sci. 8, 157 (1979).

${ }^{6}$ J. M. Kosterlitz and D. J. Thouless, J. Phys. C 6, 1181 (1973).

${ }^{7}$ D. R. Nelson and B. I. Halperin, Phys. Rev. B 19, 2457 (1979).

${ }^{8}$ A. P. Young, Phys. Rev. B 19, 1855 (1979).

${ }^{9}$ For a recent review, see K. J. Strandburg, Rev. Mod. Phys. 60, 161 (1988).

${ }^{10}$ B. J. Alder and T. E. Wainwright, Phys. Rev. 127, 359 (1962).

${ }^{11}$ W. W. Wood, in Physics of Simple Liquids, edited by H. N. Temperley, J. R. Rowlinson, and G. S. Rushbrooke (NorthHolland, Amsterdam, 1968).

${ }^{12}$ D. A. Young and B. J. Alder, J. Chem. Phys. 60, 1254 (1974).

${ }^{13}$ K. J. Strandburg, J. A. Zollweg, and G. V. Chester, Phys. Rev. B 30, 2755 (1984).
${ }^{14}$ J. D. Weeks and J. Q. Broughton, J. Chem. Phys. 78, 4197 (1983).

${ }^{15}$ N. Metropolis, A. W. Rosenbluth, M. N. Rosenbluth, A. H. Teller, and E. Teller, J. Chem. Phys. 21, 1087 (1953).

16J. A. Zollweg, G. V. Chester, and P. W. Leung, Phys. Rev. B 39, 9518 (1989).

${ }^{17}$ J. Tobochnik and G. V. Chester, Phys. Rev. B 25, 6778 (1982).

${ }^{18}$ C. E. Campbell and M. Schick, Phys. Rev. A 3, 691 (1971).

${ }^{19}$ K. S. Liu, M. H. Kalos, and G. V. Chester, Phys. Rev. B 13, 1971 (1976).

${ }^{20}$ P. A. Whitlock, G. V. Chester, and M. H. Kalos, Phys. Rev. B 38, 2418 (1988).

${ }^{21}$ D. M. Ceperley and M. H. Kalos, in Monte Carlo Methods in Statistical Physics, edited by K. Binder (Springer-Verlag, New York, 1986).

${ }^{22}$ C. Udink and J. van der Elsken, Phys. Rev. B 35, 279 (1987).

${ }^{23}$ M. P. Allen, D. Frenkel, W. Gignac, and J. P. McTague, J. Chem. Phys. 78, 4206 (1983).

${ }^{24}$ R. P. Feynman, Statistical Mechanics (Benjamin, New York, 1972); R. P. Feynman and A. R. Hibbs, Quantum Mechanics and Path Integrals (McGraw-Hill, New York, 1965); L. S. Schulman, Techniques and Applications of Path Integration 
(Wiley, New York, 1981).

${ }^{25}$ This name is borrowed from some other implementation of the PIMC method because, in our case, we do not need the Trotter formula and Eq. (8) is exact.

${ }^{26}$ D. Chandler and P. G. Wolynes, J. Chem. Phys. 74, 4078 (1981).

${ }^{27}$ See, e.g., M. Takahashi and M. Imada, J. Phys. Soc. Jpn. 53, 963 (1984); 53, 3770 (1984); F. F. Abraham and J. Q. Broughton, Phys. Rev. Lett. 59, 64 (1987); Y. J. Wong and G. V. Chester, Phys. Rev. B 37, 9590 (1988).

${ }^{28}$ J. A. Barker, J. Chem. Phys. 70, 2914 (1979).

${ }^{29} \mathrm{G}$. Jacucci, in Monte Carlo Methods in Quantum Problems, edited by M. H. Kalos (Reidel, Holland, 1984).

${ }^{30}$ E. L. Pollack and D. M. Ceperley, Phys. Rev. B 30, 2555 (1984).

${ }^{31}$ L. H. Nosanow, in Quantum Solids and Fluids, edited by S. B. Trickey, E. D. Adams, and J. W. Duffy (Plenum, New York, 1977).

${ }^{32}$ K. J. Runge and G. V. Chester, Phys. Rev. B 38, 135 (1988).

${ }^{33} \mathrm{~A}$ detailed description can be found in Y. J. Wong, Ph.D. thesis, Cornell University, 1988, App. 3.A (unpublished).

${ }^{34}$ B. Jancovici, Phys. Rev. 178, 295 (1969).

${ }^{35}$ K. E. Schmidt (private communication).

${ }^{36}$ D. M. Ceperley and E. L. Pollock, Phys. Rev. B 56, 351 (1986).

${ }^{37}$ L. D. Landau and E. M. Lifshitz, Theory of Elasticity (Pergamon, New York, 1986).

${ }^{38}$ See, e.g., N. W. Ashcroft and N. D. Mermin, Solid State Physics (Holt, New York, 1976), Chap. 23, App. L.

${ }^{39}$ G. A. Stewart, Phys. Rev. A 10, 671 (1974).

${ }^{40}$ A. A. Maradudin, E. W. Montroll, G. H. Weiss, and I. P. Ipatova, Theory of Lattice Dynamics in the Harmonic Approximation (Academic, New York, 1971).

${ }^{41}$ There is no evidence that such a mapping in two dimensions works as good as in three dimensions as in Ref. 32. We just want to get the order of magnitude of the temperature scale by analogy.

${ }^{42}$ R. A. Aziz, V. P. S. Nain, J. S. Carley, W. L. Taylor, and G. T. McConville, J. Chem. Phys. 70, 4330 (1979).

${ }^{43}$ M. H. Kalos, D. Levesque, and L. Verlet, Phys. Rev. A 9, 2178 (1974).

${ }^{44}$ X. Z. Ni and L. W. Bruch, Phys. Rev. B 33, 4584 (1986).

${ }^{45}$ Remember these are reduced densities. For the LennardJones potential, $\rho_{m} \sigma_{\mathrm{LJ}}^{2}=0.44$, where $\sigma_{\mathrm{LJ}}=2.556 \AA$. For the Aziz potential, $\rho_{m} r_{A}^{2}=0.33$, where $r_{A}=2.9673 \AA$.

${ }^{46}$ Y. J. Wong and G. V. Chester, Phys. Rev. B 35, 3506 (1987).

${ }^{47}$ The one close to melting, i.e., at $\rho=0.45$ and $T=2.0$, is really problematic in this aspect and will be discussed in the next subsection.

${ }^{48}$ Note the difference in the definitions of $A$ and $B$ in Ref. 12.

${ }^{49}$ L. D. Landau and E. M. Lifshitz, Statistical Physics, 3rd ed. (Pergamon, New York, 1989), Part I, Sec. 30.

${ }^{50}$ See, e.g., Fig. 16 in Ref. 16. There the system is much larger and is very closed to melting. It shows similar motions involving a group of particles instead of just two.

${ }^{51} \mathrm{~J}$. A. Zollweg, in Ordering in Two Dimensions, edited by S. K. Sinha (North-Holland, Amsterdam, 1980).

${ }^{52}$ J. P. McTague, D. Frenkel, and M. P. Allen, in Ordering in Two Dimensions, edited by S. K. Sinha (North-Holland, Amsterdam, 1980).

53J. A. Zollweg and G. V. Chester (unpublished).

${ }^{54}$ S. Vitiello, K. J. Runge, and M. H. Kalos, Phys. Rev. Lett. 60, 1970 (1988).

55 J. Q. Broughton, G. H. Gilmer, and J. D. Weeks, Phys. Rev. B 25, 4651 (1982).

${ }^{56}$ D. R. Squire, A. C. Holt, and W. G. Hoover, Physica (Amsterdam) 42, 388 (1968). 